\title{
Planar Dynamics of a Motorcycle: Influence of Vibration Isolation System Nonlinearity
}

\author{
Sudhir Kaul \\ School of Engineering and Technology, Western Carolina University, Cullowhee, NC, 28723, USA.
}

(Received 26 August 2020; accepted 23 September 2020)

This paper investigates the influence of the nonlinearities of a vibration isolation system on the planar dynamics of a motorcycle. The use of a nonlinear isolation system is often necessitated by design and packaging constraints. Although the use of a vibration isolation system is uncommon in motorcycles, it is used in some cases to enhance ride comfort by mitigating vibrations transmitted to the rider due to shaking forces. In such cases, the handling of the motorcycle can be influenced due to the coupled dynamics of the rear unsprung mass and the swing arm. In this paper, a stochastic analysis has been performed by using the statistical linearization method to specifically examine nonlinearities associated with the vibration isolation system. An eight degree-of-freedom planar model has been developed, and each isolator is represented by a modified multi-axial Kelvin-Voigt model. It has been observed that the model developed in this study can capture the coupled dynamics between the rear suspension and the vibration isolation system. Results indicate that the nonlinear design of the vibration isolation system can be useful in enhancing ride comfort in the lower frequency range without an adverse impact on handling. Furthermore, it has been observed that the parameters associated with the nonlinear vibration isolation system can be tuned to enhance ride comfort while meeting the design requirements of spatial dynamics.

\section{INTRODUCTION}

Planar or in-plane dynamics of a motorcycle entails the dynamics of the suspension system, wheels, frame, powertrain, and the vibration isolation system when the motorcycle is traveling in a straight line. While intertwined with spatial (or outof-plane) dynamics, planar dynamics can be specifically analyzed for ride comfort and handling. Ride comfort primarily pertains to the isolation of the rider (or sprung mass) from multiple excitation inputs while handling is a broader term that is used to convey multiple aspects of the riding experience. For planar dynamics, handling is typically used to convey the ability of the tires to adhere to the road surface. Multi-body dynamics models are being increasingly used to analyze the influence of several components or configurations on stability, handling, maneuverability, etc. of a motorcycle. Availability of commercial software such as BikeSim and FastBike enables designers to investigate dynamic characteristics before commencing detailed design. ${ }^{1,2}$ While the multi-body dynamics models developed in commercial software are useful for a system level analysis, their use is somewhat limited for component level design of parts such as vibration isolators. Furthermore, most of the multi-body dynamics models assume linear behavior and ignore any nonlinearities. Some of the research in motorcycle dynamics has focused on modeling special purpose motorcycles that are used for off-road or racing purposes. These systems require modeling adjustments since road irregularities could induce high amplitude displacements that may result in a nonlinear response. ${ }^{3}$

Nonlinear designs of vibration isolators are useful in mitigating some of the inherent trade-offs associated with the design of linear and passive systems. ${ }^{4}$ In some cases, nonlinear designs incorporate characteristics such as quasi-zero stiffness (QZS) and high-static-low-dynamic stiffness (HSLDS). Although the introduction of such nonlinearities is helpful in reducing some of the design trade-offs between resonant peaks and high frequency transmissibility, such designs often result in phenomena such as instability, jump frequencies, hardening or softening behavior, etc., that may need to be carefully examined and simulated. ${ }^{4-7}$ Incorporation of stiffness nonlinearity enables a vibration isolation system to exhibit a significantly higher stiffness along the non-isolating axes. This is important for some products in automotive systems where packaging, handling, etc. are critical considerations in the design of a vibration isolation system. ${ }^{8}$ Such constraints require a vibration isolator to demonstrate significantly different stiffness and damping properties along the isolating axis as compared to the other axes. One example of such a vibration isolator is shown in Fig. 1. In addition to the design nonlinearity of the vibration isolator model shown in Fig. 1, there are several other nonlinearities associated with different designs of a vibration isolator. In the literature, the study of such nonlinearities includes investigation of the influence of cubic damping, ${ }^{9}$ material properties of rubber, ${ }^{10}$ and damping mechanisms, ${ }^{11}$ among other viscoelastic characteristics. ${ }^{12}$

Viscoelastic models are commonly used for modeling elastomeric isolators in order to represent specific properties involving damping and stiffness characteristics. The main advantage of these models is a rheological relationship between multiple physical parameters of the vibration isolator and the viscoelastic model, allowing design iterations that can relate attributes such as isolator geometry and elastomeric material properties to specific aspects of the system response. Such models are often used to represent the complex behavior of an elastomer to perform dynamic simulations, ${ }^{13}$ or for the analysis or optimization of the dynamic response of an elastomeric isolation system. ${ }^{14}$

A vibration isolation system is not commonly used in motorcycles. However, the use of such a system is necessary in some cases to mitigate the effect of shaking forces resulting 


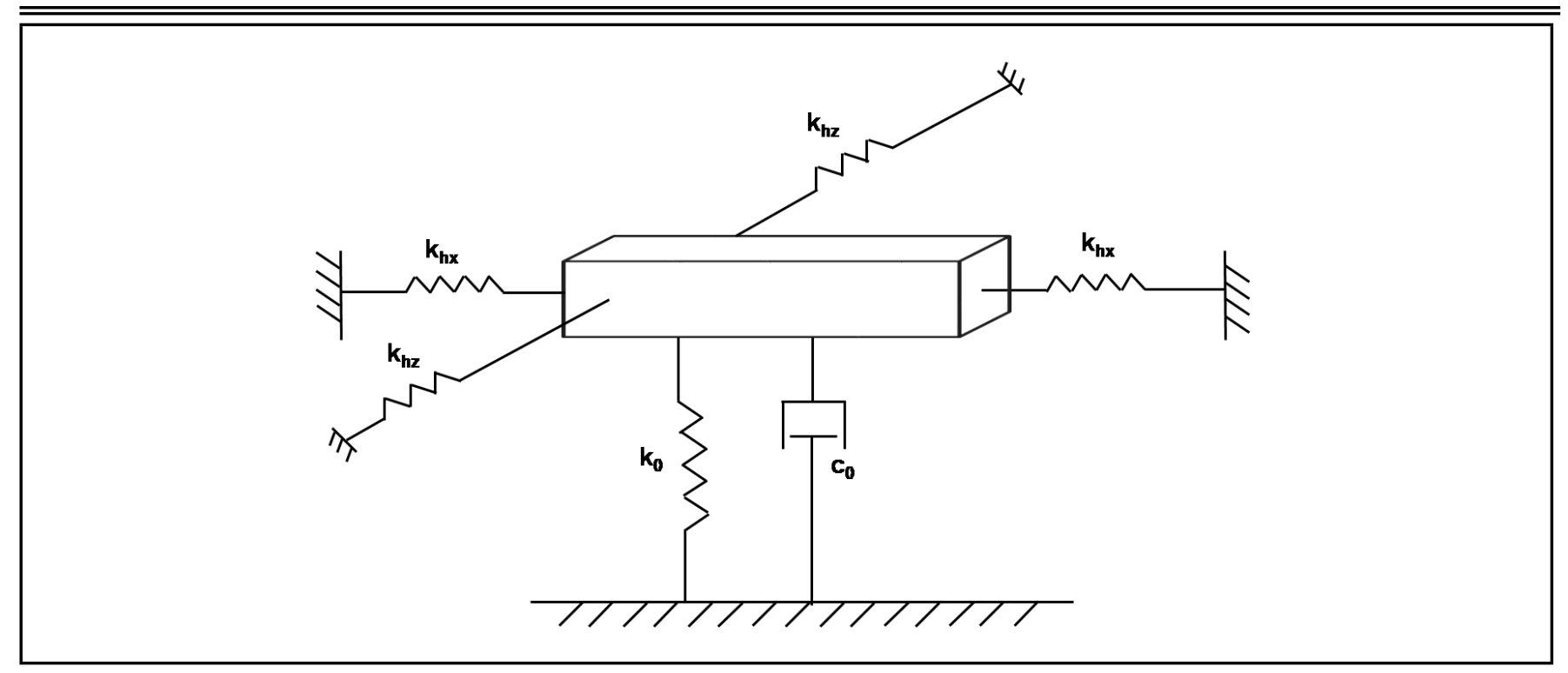

Figure 1. Modified nonlinear Kelvin-Voigt model.

from the configuration of the powertrain and other packaging constraints. These shaking forces result in vibrations that are felt by the rider at the seat, handlebars, and footrests, typically at relatively higher frequencies. Although the primary purpose of the vibration isolation system is to enhance ride comfort, its inherent coupling with the rear suspension and the rear unsprung mass influences the overall planar, and arguably spatial, dynamics of the motorcycle. ${ }^{15}$ This makes the design of the vibration isolation system challenging and requires a careful examination of design parameters such as stiffness nonlinearity, material damping, etc. in order to mitigate the trade-off between ride comfort and handling. Although planar dynamics of motorcycles has been investigated in the existing literature, there is no model in the current literature that captures the influence of the vibration isolation system, and its associated nonlinearities, on ride comfort and handling. This paper attempts to fill this gap by providing a comprehensive analytical model that can be used to investigate nonlinearities of the vibration isolation system in conjunction with the suspension system in order to comprehend the influence on the planar dynamics of the motorcycle. The aim of the model presented in this paper is to provide a tool that can be used for a holistic analysis of the design parameters of the vibration isolation system to comprehend their influence on ride comfort as well as handling.

In this paper, Section 2 presents the proposed model as well as the analysis method used in this study. This section includes a brief description of the statistical linearization method that has been used to solve the nonlinear model. Section 3 presents the results from multiple simulations carried out by using the model from Section 2 while comparing the results of the nonlinear model to a corresponding linear model. Overall conclusions from the results of the study are drawn in Section 4.

\section{MODEL}

The governing planar motorcycle model is presented in this section along with the assumptions used to derive the equations of motion (EOM). The model consists of four rigid bodies that are interconnected either through the suspension system or the vibration isolation system. The vibration isolator model used in this study is similar to a HSLDS design that has been discussed in recent literature, ${ }^{6,16}$ and one such design is shown in Fig. 1. The governing model of such a design has been derived in the literature for a multi-axial vibration isolator, ${ }^{8}$ and will be incorporated into the planar motorcycle model derived for this study. For the planar model discussed in this section, each suspension system is represented as a spring-damper unit. ${ }^{17}$ The entire model is shown in Fig. 2.

In the model shown in Fig. 2, the chassis and the rider are combined as one rigid body, designated the sprung mass. The sprung mass is modeled with two degrees-of-freedom (DOF), namely bounce and pitch. There are two unsprung masses in the model, one for the front wheel and one for the rear wheel. Each unsprung mass is the combined mass of the wheel and other parts connected to the wheel. While the front unsprung mass has been modeled with only one DOF, the rear unsprung mass has been modeled with two translational DOF since it is attached to the powertrain through the swing arm, as shown in the schematic of the model in Fig. 2. The powertrain has been modeled as a rigid body with two translational DOF and one rotational or pitch DOF. The powertrain is connected to the sprung mass through two sets of vibration isolators and to the rear unsprung mass through the swing arm (also known as the rear fork in the literature). The swing arm is typically attached through a revolute or pin joint and connects the rear wheel to the frame as well as the powertrain. ${ }^{16}$ The EOM of all four rigid bodies are combined to identify the overall mass $(M)$, damping $(C)$ and stiffness $(K)$ matrices of the system, all three matrices are $8 \times 8$. The mass matrix is diagonal due to the lumped mass representation of all the rigid bodies in the model. The linear model can be directly referenced from the literature and is briefly summarized in the following subsection before discussing the nonlinear model. ${ }^{15}$

\subsection{Linear Model}

The linear model uses a conventional Kelvin-Voigt model for each vibration isolator connecting the powertrain to the sprung mass. The EOM for this model can be expressed in the matrix form as:

$$
M \ddot{Y}+C \dot{Y}+K Y=K^{\prime \prime} X+C^{\prime \prime} \dot{X} .
$$




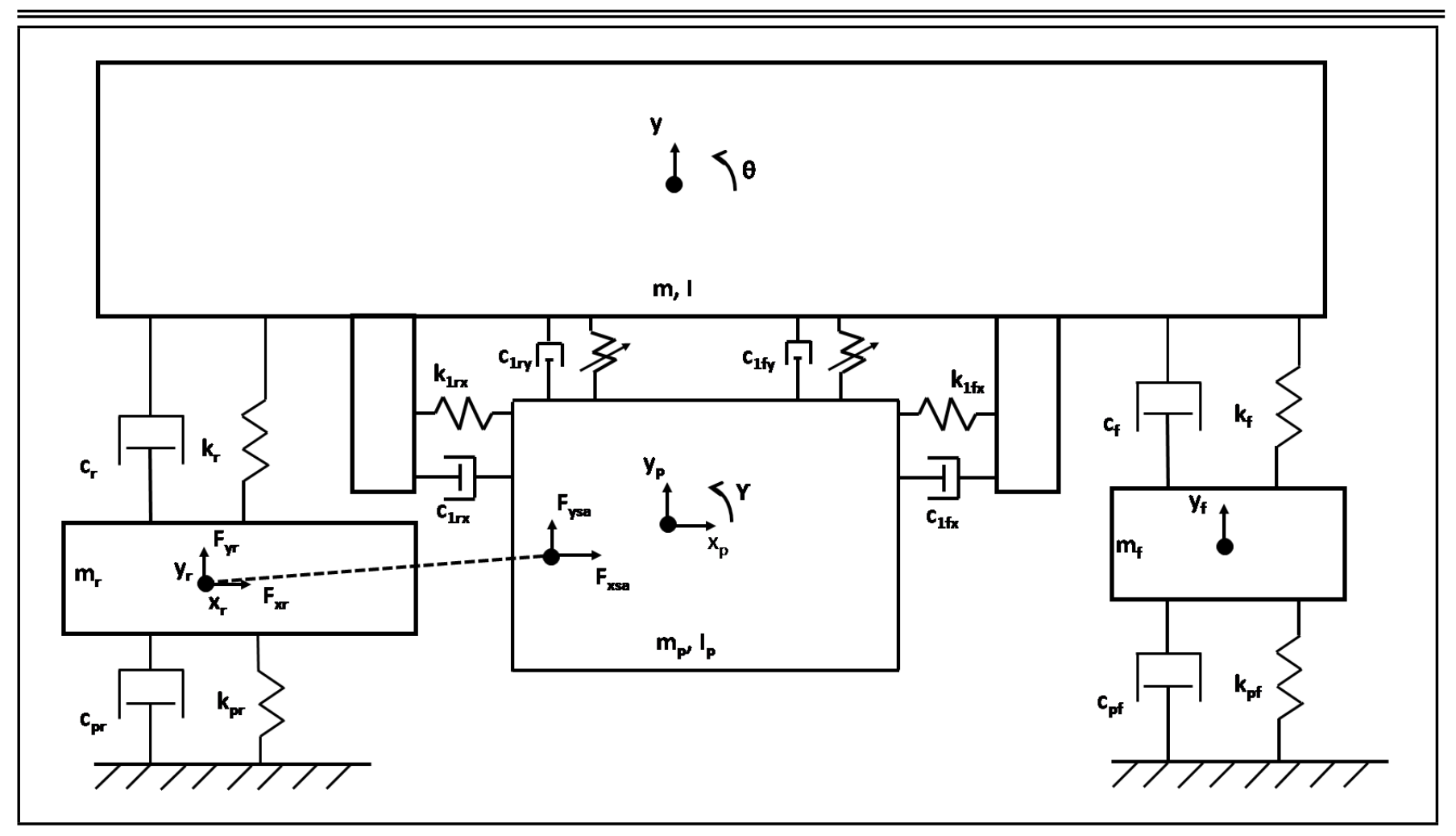

Figure 2. Eight degree-of-freedom model including isolator nonlinearities.

In Eq. (1), $Y=\left[\begin{array}{lllllll}y & \theta & y_{f} & x_{r} & y_{r} & x_{p} & y_{p}\end{array}\right]^{T}$ is the $8 \times 1$ vector that consists of the eight DOF located at the center of mass of each rigid body. $K^{\prime \prime}$ and $C^{\prime \prime}$ are $8 \times 2$ matrices resulting from the source of base excitation at the front and rear wheels, with $K^{\prime \prime}=\left[\begin{array}{llllllllllllllll}0 & 0 & k_{p f} & 0 & 0 & 0 & 0 & 0 ; & 0 & 0 & 0 & 0 & k_{p r} & 0 & 0 & 0\end{array}\right]^{T}$ and $C^{\prime \prime}=\left[\begin{array}{lllllllllllllll}0 & 0 & c_{p f} & 0 & 0 & 0 & 0 & 0 ; & 0 & 0 & 0 & 0 & c_{p r} & 0 & 0\end{array}\right]^{T}$, where $k_{p f}$ and $c_{p f}$ are the stiffness and damping constants of the front tire while $k_{p r}$ and $c_{p r}$ are the stiffness and damping constants of the rear tire. In Eq. (1), $X$ is the base excitation at the tires due to irregularity of the road surface over which the motorcycle is traveling, $X=\left[\begin{array}{ll}y_{f i}(t) & y_{f i}\left(t-\frac{p}{V}\right)\end{array}\right]^{T}$. This base excitation accounts for wheelbase filtering due to the lag in excitation between the front tire and the rear tire as the motorcycle travels at a constant velocity, $V$, in a straight line with a wheelbase of $p$, and with a vertical displacement amplitude of $y_{f i}$ due to road irregularity at the front wheel of the motorcycle.

The acceleration response, $A(\omega)$, for the model in Eq. (1), can be expressed as follows:

$$
A(\omega)=-\omega^{2}\left[-\omega^{2} M+i \omega C+K\right]^{-1}\left[K^{\prime \prime}+i \omega C^{\prime \prime}\right] X(\omega) .
$$

In Eq. (2), $X(\omega)=\left[\begin{array}{c}1 \\ e^{-\omega \frac{p}{V}}\end{array}\right] Y_{f i}(\omega)$, where $Y_{f i}(\omega)$ is the input resulting from the irregularity of the road surface at the front tire in the frequency domain, this form of the input is due to the effect of wheelbase filtering. The acceleration response of Eq. (2) can be modified as:

$$
A(\omega)=H(\omega)\left[\begin{array}{c}
1 \\
e^{-\omega \frac{p}{V}}
\end{array}\right] Y_{f i}(\omega)=H^{*}(\omega, V) Y_{f i}(\omega)
$$

In Eq. (3), $H(\omega)=-\omega^{2}\left[-\omega^{2} M+i \omega C+K\right]^{-1}\left[K^{\prime \prime}+i \omega C^{\prime \prime}\right]$, and $H^{*}(\omega, V)$ is the $8 \times 1$ modified frequency response function (FRF) that can be used to compute the power spectral density (PSD) or spectrum of acceleration for each DOF of the model as follows:

$$
S_{a}(\omega, V)=\left|H_{a}^{*}(\omega, V)\right|^{2} S_{r r}(\omega, V) .
$$

In Eq. (4), $S_{r r}(\omega, V)$ is the PSD of the road profile. Furthermore, $S_{a}(\omega, V)$ is the PSD of acceleration of each DOF, where $a=\ddot{y}, \ddot{\theta}, \ddot{y}_{f}, \ddot{x}_{r}, \ddot{y}_{r}, \ddot{x}_{p}, \ddot{y}_{p}, \ddot{\gamma}$, and is computed by using the magnitude of the modified FRF, $H_{a}^{*}(\omega, V)$, for each degreeof-freedom. For this study, the PSD of the road profile has been computed by assuming a constant speed of the motorcycle while it is traveling on a good road surface. ${ }^{19}$ The details of this linear model can be found in the literature. ${ }^{15}$

\subsection{Nonlinear Model}

In order to account for the nonlinearity introduced by the multi-axial stiffness of the vibration isolators, as shown in Fig. 1, the restoring spring force due to the effective vertical stiffness of the front and rear isolators $\left(f_{1 f y}\right.$ and $f_{1 r y}$ respectively) can be expressed as follows: ${ }^{8}$

$$
f_{1 f y}=f_{1 r y}=\beta_{2} y_{1}+\beta_{1} y_{1}^{3} .
$$

In Eq. (5), $y_{1}$ is the relative displacement of the rigid body in the vertical direction while $\beta_{1}$ and $\beta_{2}$ are coefficients dependent on stiffness, free length of the spring elements, and the compressed length of the spring elements at static equilibrium. It may be noted that the front and rear isolators are assumed to be identical to simplify the model even though the relative displacement for each isolator is expected to be different. The model as well as the analysis presented in this paper is not limited to identical front and rear isolators, and can be adapted to a varying number of isolators with different characteristics. In Eq. (5), $\beta_{1}=k_{h x} \frac{l_{0 x}}{l_{x}^{3}}+k_{h z} \frac{l_{0 z}}{l_{z}^{3}}$ and $\beta_{2}=k_{0}+2 k_{h x}-2 k_{h x} \frac{l_{0 x}}{l_{x}}+2 k_{h z}-2 k_{h z} \frac{l_{0 z}}{l_{z}}$, where the free length of the two springs along the $x$-axis and the $z$-axis is $l_{0 x}$ 
and $l_{0 z}$ respectively while the compressed length upon assembly, at static equilibrium, is $l_{x}$ and $l_{z}$. Also, $k_{0}, k_{h x}, k_{h z}$ are the stiffness constants as shown in the model in Fig. 1. The two isolators (front and rear) are assumed to be identical, and in order to further simplify the model, the following substitutions have been used for the two identical nonlinear vibration isolators: $k_{h x}=k_{h z}=k_{h}, l_{0 x}=l_{0 z}=l_{0}, l_{x}=l_{z}=l$. As a result, $\beta_{1}=2 k_{h} \frac{l_{0}}{l^{3}}$ and $\beta_{2}=k_{0}+4 k_{h}-4 k_{h} \frac{l_{0}}{l^{3}}$ can be substituted in Eq. (5). The benefit of such an isolator design can be visualized by plotting the progressive stiffness exhibited by the vibration isolator along the vertical axis. The effective stiffness, $k_{\text {eff }}$, of each vibration isolator in the vertical direction can be derived as:

$$
k_{\mathrm{eff}}=\frac{\partial f_{1 f y}}{\partial y_{1}}=\frac{\partial f_{1 r y}}{\partial y_{1}}=\beta_{2}+3 \beta_{1} y_{1}^{2}
$$

In Eq. (6), $y_{1}$ is the relative displacement along the isolation direction. It may be noted that stability can be ensured by choosing the parameters such that $\beta_{2}>0 .{ }^{16}$ For the design used in this study, a pre-compression of $5 \%$ has been used unless otherwise stated, resulting in $l=0.95 l_{0}$. Also, due to the selection of $5 \%$ pre-compression, $k_{h}=4 k_{0}$ has been enforced for satisfying the stability criterion. The resulting progressive stiffness of this vibration isolator along the vertical axis can be seen in Fig. 3 for a free length, $l_{0}$, of $50 \mathrm{~mm}$ and varying values of $k_{0}$. As can be seen from Fig. 3, the effective stiffness is progressive for the three values of $k_{0}$ shown in the figure as compared to the fixed value of stiffness, $k=750 \mathrm{~N} / \mathrm{mm}$, for a comparable linear vibration isolator used for the linear model. It may be noted that the choice of model parameters for the vibration isolators that has been discussed here will ensure that the system will not exhibit instabilities or jump frequencies for the analysis performed in this paper.

The EOM in Eq. (1) need to be modified to accommodate the nonlinearities due to the influence of the multi-axial stiffness of the vibration isolators connecting the powertrain to the sprung mass. The corresponding EOM for the sprung mass are derived to be as follows:

$$
\begin{aligned}
& m \ddot{y}+\left(k_{f}+k_{r}+2 \beta_{2}\right) y-k_{f} y_{f}-k_{r} y_{r}+ \\
& {\left[k_{f}(p-b)-k_{r} b+\beta_{2} x_{f 1}-\beta_{2} x_{r 1}\right] \theta+\left(\beta_{2} x_{r 1}-\beta_{2} x_{f 1}\right) \gamma-} \\
& 2 \beta_{2} y_{p}+\left(c_{f}+c_{r}+c_{1 f y}+c_{1 r y}\right) \dot{y}-c_{f} \dot{y}_{f}-c_{r} \dot{y}_{r}+ \\
& {\left[c_{f}(p-b)-c_{r} b+c_{1 f y} x_{f 1}-c_{1 r y} x_{r 1}\right] \dot{\theta}+} \\
& \left(c_{1 r y} x_{r 1}-c_{1 f y} x_{f 1}\right) \dot{\gamma}+\left(-c_{1 f y}-c_{1 r y}\right) \dot{y}_{p}- \\
& \beta_{1}\left(y_{p}+x_{f 1} \gamma-y-x_{f 1} \theta\right)^{3}- \\
& \beta_{1}\left(y_{p}-x_{r 1} \gamma-y+x_{r 1} \theta\right)^{3}=0
\end{aligned}
$$

$$
\begin{aligned}
& I \ddot{\theta}+\left[k_{f}(p-b)^{2}+k_{r} b^{2}+\beta_{2} x_{f 1}^{2}+\beta_{2} x_{r 1}^{2}\right] \theta+ \\
& {\left[k_{f}(p-b)-k_{r} b+\beta_{2} x_{f 1}-\beta_{2} x_{r 1}\right] y-k_{f}(p-b) y_{f}+} \\
& k_{r} b y_{r}+\left(\beta_{2} x_{r 1}-\beta_{2} x_{f 1}\right) y_{p}+\left(-\beta_{2} x_{f 1}^{2}-\beta_{2} x_{r 1}^{2}\right) \gamma+ \\
& {\left[c_{f}(p-b)^{2}+c_{r} b^{2}+c_{1 f y} x_{f 1}^{2}+c_{1 r y} x_{r 1}^{2}\right] \dot{\theta}+} \\
& {\left[c_{f}(p-b)-c_{r} b+c_{1 f y} x_{f 1}-c_{1 r y} x_{r 1}\right] \dot{y}-c_{f}(p-b) \dot{y}_{f}+} \\
& c_{r} b \dot{y}_{r}+\left(c_{1 r y} x_{r 1}-c_{1 f y} x_{f 1}\right) \dot{y}_{p}+\left(-c_{1 f y} x_{f 1}^{2}-c_{1 r y} x_{r 1}^{2}\right) \dot{\gamma}- \\
& \beta_{1} x_{f 1}\left(y_{p}+x_{f 1} \gamma-y-x_{f 1} \theta\right)^{3}+ \\
& \beta_{1} x_{r 1}\left(y_{p}-x_{r 1} \gamma-y+x_{r 1} \theta\right)^{3}=0 .
\end{aligned}
$$

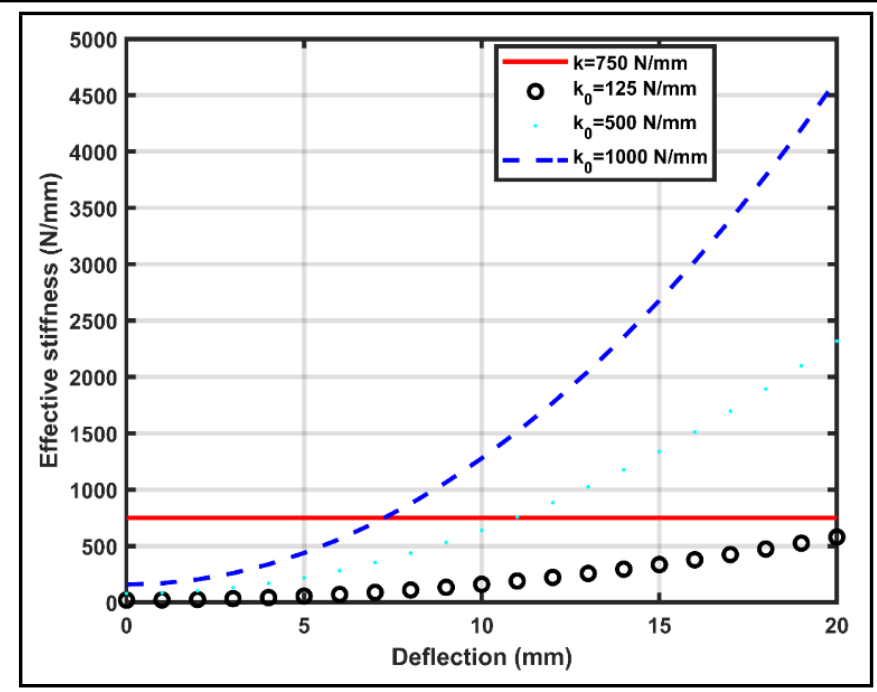

Figure 3. Nonlinear stiffness characteristics.

In Eq. (7) and Eq. (8), $m$ and $I$ are the mass and mass moment of inertia about the center of mass of the sprung mass. In the EOM, $y$ represents bounce (vertical motion) and $\theta$ represents the pitch motion of the sprung mass while $x_{p}, y_{p}$, and $\gamma$ represent the motion of the powertrain. The location of the elastic center of the two vibration isolators from the center of mass of the powertrain is $\left(x_{f 1}, y_{f 1}\right)$ and $\left(x_{r 1}, y_{r 1}\right)$. Although the distribution of the rider mass and inertia as well as the rider position can influence the dynamics of the motorcycle, the model presented in this study does not account for this variability. As a result, the rider mass and inertia in an upright position is combined with the chassis mass and inertia. In Eq. (7) and Eq. (8), the powertrain is assembled to the frame (sprung mass) through vibration isolators with damping constants of $\left(c_{1 f x}, c_{1 f y}\right)$ and $\left(c_{1 r x}, c_{1 r y}\right)$ at the front and rear, and horizontal stiffness constants of $k_{1 f x}$ and $k_{1 r x}$ at the front and rear respectively. Also, in Eq. (7) and Eq. (8), $\beta_{1}$ and $\beta_{2}$ are the coefficients associated with the multi-axial vibration isolators discussed in Eq. (5) and Eq. (6). It may be noted that the EOM have been derived for a wheelbase of $p$, and the distance between the center of mass of the sprung mass and the center of mass of the rear unsprung mass is expressed as $b$.

The EOM for the two unsprung masses are derived to be as:

$$
\begin{gathered}
m_{f} \ddot{y}_{f}-k_{f} y-k_{f}(p-b) \theta+\left(k_{f}+k_{p f}\right) y_{f}- \\
c_{f} \dot{y}-c_{f}(p-b) \dot{\theta}+\left(c_{f}+c_{p f}\right) \dot{y}_{f}=0 ; \\
m_{r} \ddot{x}_{r}=F_{x r} \\
m_{r} \ddot{y}_{r}+\left(k_{p r}+k_{r}\right) y_{r}-k_{r} y+k_{r} b \theta+ \\
\left(c_{p r}+c_{r}\right) \dot{y}_{r}-c_{r} \dot{y}+c_{r} b \dot{\theta}=F_{y r} .
\end{gathered}
$$

As can be seen from Eq. (9), Eq. (10) and Eq. (11), the hop (vertical) DOF associated with both unsprung masses ( $y_{r}$ and $\left.y_{f}\right)$ is modeled while the fore-aft DOF of the rear unsprung mass $\left(x_{r}\right)$ is modeled additionally due to the attachment of the rear unsprung mass to the powertrain through the swing arm. In Eq. (10) and Eq. (11), $F_{x r}$ and $F_{y r}$ are the fore-aft and vertical components of the force resulting from the pin connection between the swing arm and the rear unsprung mass. Furthermore, $m_{f}$ and $m_{r}$ are the front and rear unsprung masses while $k_{f}$ and $k_{r}$ are the effective spring constants for the front and rear suspensions. 
The powertrain is modeled with three planar DOF and the corresponding EOM are derived as follows:

$$
\begin{aligned}
& m_{p} \ddot{x}_{p}+\left(k_{1 f x}+k_{1 r x}\right) x_{p}+\left(k_{1 r x} y_{r 1}-k_{1 f x} y_{f 1}\right) \gamma+ \\
& \left(c_{1 f x}+c_{1 r x}\right) \dot{x}_{p}+\left(c_{1 r x} y_{r 1}-c_{1 f x} y_{f 1}\right) \dot{\gamma}=F_{x s a} \\
& m_{p} \ddot{y}_{p}+2 \beta_{2} y_{p}+\left(\beta_{2} x_{f 1}-\beta_{2} x_{r 1}\right) \gamma+ \\
& \left(-\beta_{2} x_{f 1}+\beta_{2} x_{r 1}\right) \theta-2 \beta_{2} y+\left(c_{1 f y}+c_{1 r y}\right) \dot{y}_{p}+ \\
& \left(c_{1 f y} x_{f 1}-c_{1 r y} x_{r 1}\right) \dot{\gamma}+\left(-c_{1 f y} x_{f 1}+c_{1 r y} x_{r 1}\right) \dot{\theta}+ \\
& \left(-c_{1 f y}-c_{1 r y}\right) \dot{y}+\beta_{1}\left(y_{p}+x_{f 1} \gamma-y-x_{f 1} \theta\right)^{3}+ \\
& \beta_{1}\left(y_{p}-x_{r 1} \gamma-y+x_{r 1} \theta\right)^{3}=F_{y s a} ;
\end{aligned}
$$

$$
\begin{aligned}
& I_{p} \ddot{\gamma}+\left(k_{1 f x} y_{f 1}^{2}+\beta_{2} x_{f 1}^{2}+k_{1 r x} y_{r 1}^{2}+\beta_{2} x_{r 1}^{2}\right) \gamma+ \\
& \left(k_{1 r x} y_{r 1}-k_{1 f x} y_{f 1}\right) x_{p}+\left(\beta_{2} x_{f 1}-\beta_{2} x_{r 1}\right) y_{p}+ \\
& \left(-\beta_{2} x_{f 1}+\beta_{2} x_{r 1}\right) y+\left(-\beta_{2} x_{f 1}^{2}-\beta_{2} x_{r 1}^{2}\right) \theta+ \\
& \left(c_{1 f x} y_{f 1}^{2}+c_{1 f y} x_{f 1}^{2}+c_{1 r x} y_{r 1}^{2}+c_{1 r y} x_{r 1}^{2}\right) \dot{\gamma}+ \\
& \left(c_{1 r x} y_{r 1}-c_{1 f x} y_{f 1}\right) \dot{x}_{p}+\left(c_{1 f y} x_{f 1}-c_{1 r y} x_{r 1}\right) \dot{y}_{p}+ \\
& \left(-c_{1 f y} x_{f 1}+c_{1 r y} x_{r 1}\right) \dot{y}+\left(-c_{1 f y} x_{f 1}^{2}-c_{1 r y} x_{r 1}^{2}\right) \dot{\theta}+ \\
& \beta_{1} x_{f 1}\left(y_{p}+x_{f 1} \gamma-y-x_{f 1} \theta\right)^{3}- \\
& \beta_{1} x_{r 1}\left(y_{p}-x_{r 1} \gamma-y+x_{r 1} \theta\right)^{3}=F_{x s a} y_{s a}-F_{y s a} x_{s a} .
\end{aligned}
$$

In Eqs. (12)-(14), $m_{p}$ and $I_{p}$ are the mass and mass moment of inertia of the powertrain while $x_{p}, y_{p}$, and $\gamma$ represent the three DOF of the powertrain. Additionally, $\left(x_{s a}, y_{s a}\right)$ is the location of the swing arm pivot on the powertrain from its center of mass. It may be noted that $x_{s a}$ and $y_{s a}$ are the absolute values of the coordinates. In Eqs. (12)-(14), $F_{x s a}$ and $F_{y s a}$ are the horizontal and vertical components of the force resulting from the pin connection between the swing arm and the powertrain. Since the swing arm is attached through a pin joint at both ends, it has been modeled as a one-dimensional uniaxial spring. Furthermore, since the swing arm is typically designed to be highly stiff, such a representation is expected to be adequate for the planar model. Using this model, the connection forces at the two ends of the swing arm are derived as:

$$
\begin{aligned}
F_{x r}= & \frac{A E}{L}\left[x_{r} \cos ^{2} \phi+y_{r} \cos \phi \sin \phi-\right. \\
& \left.\left(x_{p}+y_{s a} \gamma\right) \cos ^{2} \phi-\left(y_{p}-x_{s a} \gamma\right) \cos \phi \sin \phi\right] ; \\
F_{y r}= & \frac{A E}{L}\left[y_{r} \sin ^{2} \phi+x_{r} \cos \phi \sin \phi-\right. \\
& \left.\left(x_{p}+y_{s a} \gamma\right) \cos \phi \sin \phi-\left(y_{p}-x_{s a} \gamma\right) \sin ^{2} \phi\right] ; \\
F_{x s a}= & \frac{A E}{L}\left[-x_{r} \cos ^{2} \phi-y_{r} \cos \phi \sin \phi+\right. \\
& \left.\left(x_{p}+y_{s a} \gamma\right) \cos ^{2} \phi+\left(y_{p}-x_{s a} \gamma\right) \cos \phi \sin \phi\right] ; \\
F_{y s a}= & \frac{A E}{L}\left[-y_{r} \sin ^{2} \phi-x_{r} \cos \phi \sin \phi+\right. \\
& \left.\left(x_{p}+y_{s a} \gamma\right) \cos \phi \sin \phi+\left(y_{p}-x_{s a} \gamma\right) \sin ^{2} \phi\right] .
\end{aligned}
$$

In Eqs. (15)-(18), $A$ is the effective cross-sectional area of the swing arm, $E$ is the modulus of elasticity of the swing arm material, and $L$ is the length between the swing arm pivot on the powertrain and the pivot on the unsprung mass. Furthermore, $\phi$ is the angle of inclination of the swing arm from the horizontal axis that depends on the overall trim and may change somewhat with the hop of the rear unsprung mass. The rest of the variables in Eqs. (15)-(18) have already been defined.

The EOM in Eq. (7) to Eq. (18) are combined to express the overall nonlinear model in a matrix form that is similar to the linear model in Eq. (1), this model can be expressed as:

$$
M \ddot{Y}+C \dot{Y}+K^{*} Y+g(Y)=K^{\prime \prime} X+C^{\prime \prime} \dot{X} .
$$

In Eq. (19), the mass and damping ( $M$ and $C$ respectively) matrices are identical to the linear model in Eq. (1), the stiffness matrix, $K^{*}$, is modified to accommodate the nonlinear vibration isolator design while $g(Y)$ is the $8 \times 1$ vector containing all the terms resulting from the stiffness nonlinearity of the vibration isolators in the model. These nonlinearities are collected together in the vector form as follows:

$$
\begin{aligned}
& g(Y)= \\
& {\left[\begin{array}{c}
-\beta_{1}\left(y_{p}+x_{f 1} \gamma-y-x_{f 1} \theta\right)^{3}-\beta_{1}\left(y_{p}-x_{r 1} \gamma-y+x_{r 1} \theta\right)^{3} \\
0 \\
0 \\
0 \\
0 \\
0 \\
\beta_{1}\left(y_{p}+x_{f 1} \gamma-y-x_{f 1} \theta\right)^{3}+\beta_{1}\left(y_{p}-x_{r 1} \gamma-y+x_{r 1} \theta\right)^{3} \\
\beta_{1} x_{f 1}\left(y_{p}+x_{f 1} \gamma-y-x_{f 1} \theta\right)^{3}-\beta_{1} x_{r 1}\left(y_{p}-x_{r 1} \gamma-y+x_{r 1} \theta\right)^{3}
\end{array}\right] .}
\end{aligned}
$$

The clear separation of the terms associated with nonlinearities, as shown in the vector in Eq. (20), simplifies the use of the statistical linearization method that has been used for solving the nonlinear model.

The statistical linearization method has been used in the literature to derive an equivalent linear model that can be used to replace a nonlinear model with reasonable accuracy when the excitation input is stationary and Gaussian. ${ }^{3,20}$ In some cases, this method has also been shown to be effective for nonstationary excitation inputs. ${ }^{20}$ The determination of the equivalent linear model is based on statistically minimizing the Euclidean norm of the error. For spectral response, the error is computed as the difference between the covariances of the acceleration spectrum resulting from the iterative calculation of the system response. The components of the equivalent linear system can be computed by using an iterative process and a corresponding convergence criterion. ${ }^{20}$ This iterative process has been used to replace the EOM in Eq. (19) with an equivalent linear model that can be expressed as:

$\left(M+M_{e}\right) \ddot{Y}+\left(C+C_{e}\right) \dot{Y}+\left(K^{*}+K_{e}\right) Y=K^{\prime \prime} X+C^{\prime \prime} \dot{X}$.

In Eq. (21), $M_{e}, C_{e}$, and $K_{e}$ are the equivalent mass, damping, and stiffness matrices derived to replace the nonlinear system with an equivalent linear system. Since all the nonlinearities in the model presented in this paper are limited to the stiffness matrix, $M_{e}$ and $C_{e}$ are zero matrices for the EOM derived for the model in Fig. 2. It may be noted that the statistical linearization method can also be used to deal with other forms of nonlinearities for the model discussed in this paper. In such cases, the mass matrix, $M_{e}$, and the damping matrix, $C_{e}$, may be non-zero, depending on the nature of system nonlinearities.

The elements of the stiffness matrix, $K_{e}$, in Eq. (21) can be derived from the vector containing the nonlinear terms, $g(Y)$, shown in Eq. (20), as $K_{i j}^{e}=E\left[\frac{\partial g_{i}}{\partial Y}\right], i=1, \ldots, 8 ; j=$ 


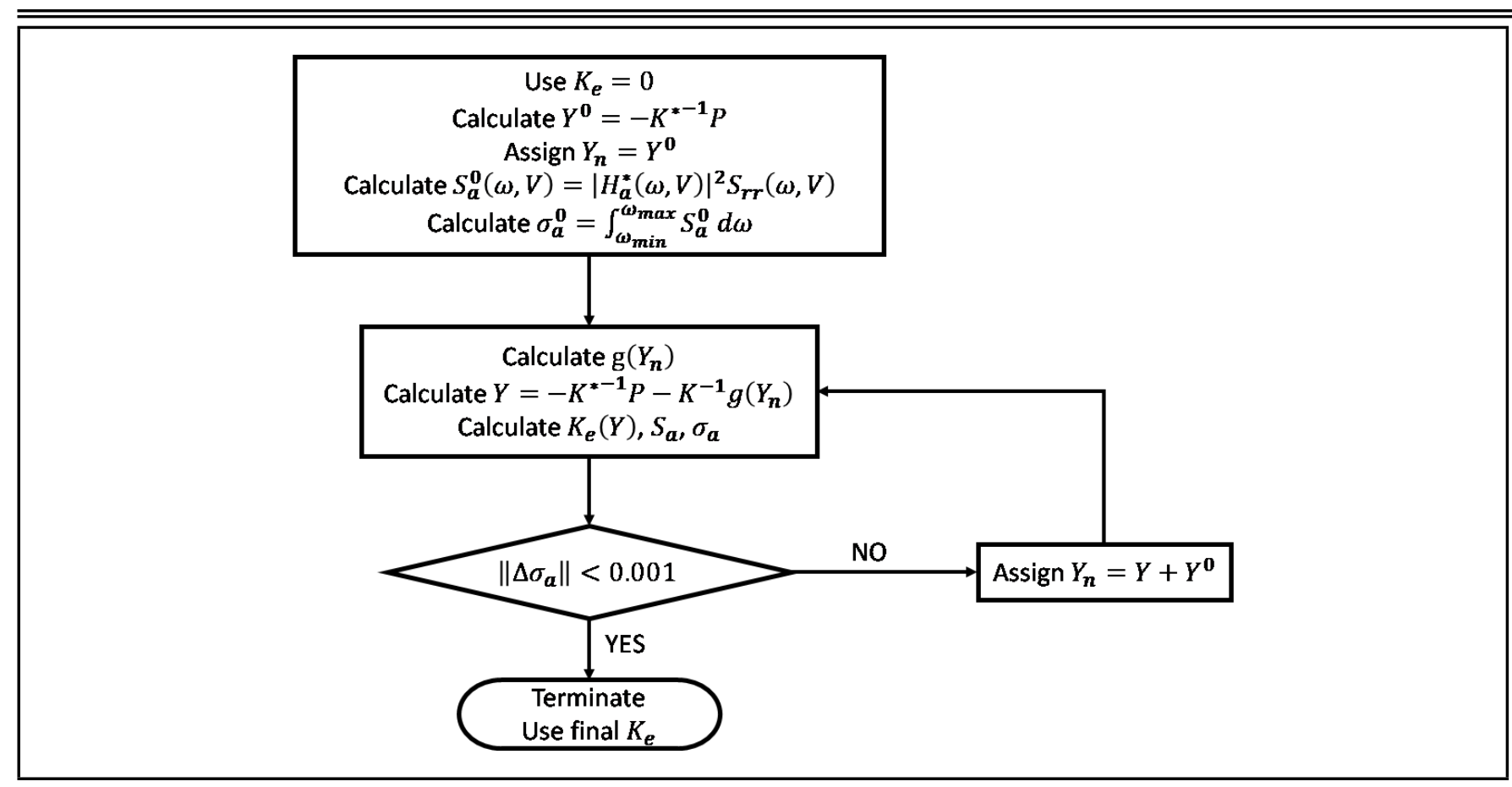

Figure 4. Statistical linearization method-algorithm.

$1, \ldots, 8$, where $Y$ is the vector consisting of all DOF of the system and $E[\cdot]$ is the expected value. These elements of the stiffness matrix, $K_{e}$, are derived row-by-row as follows:

$$
\begin{aligned}
& \frac{\partial g_{1}}{\partial Y}= \\
& {\left[\begin{array}{c}
3 \beta_{1}\left(y_{p}+x_{f 1} \gamma-y-x_{f 1} \theta\right)^{2}+3 \beta_{1}\left(y_{p}-x_{r 1} \gamma-y+x_{r 1} \theta\right)^{2} \\
3 \beta_{1} x_{f 1}\left(y_{p}+x_{f 1} \gamma-y-x_{f 1} \theta\right)^{2}-3 \beta_{1} x_{r 1}\left(y_{p}-x_{r 1} \gamma-y+x_{r 1} \theta\right)^{2} \\
0 \\
0 \\
0 \\
0 \\
-3 \beta_{1}\left(y_{p}+x_{f 1} \gamma-y-x_{f 1} \theta\right)^{2}-3 \beta_{1}\left(y_{p}-x_{r 1} \gamma-y+x_{r 1} \theta\right)^{2} \\
-3 \beta_{1} x_{f 1}\left(y_{p}+x_{f 1} \gamma-y-x_{f 1} \theta\right)^{2}+3 \beta_{1} x_{r 1}\left(y_{p}-x_{r 1} \gamma-y+x_{r 1} \theta\right)^{2}
\end{array}\right] ;}
\end{aligned}
$$$$
\frac{\partial g_{2}}{\partial Y}=\frac{\partial g_{3}}{\partial Y}=\frac{\partial g_{4}}{\partial Y}=\frac{\partial g_{5}}{\partial Y}=\frac{\partial g_{6}}{\partial Y}=0_{1 \times 8}
$$

$\frac{\partial g_{7}}{\partial Y}=$

$\left[\begin{array}{c}-3 \beta_{1}\left(y_{p}+x_{f 1} \gamma-y-x_{f 1} \theta\right)^{2}-3 \beta_{1}\left(y_{p}-x_{r 1} \gamma-y+x_{r 1} \theta\right)^{2} \\ -3 \beta_{1} x_{f 1}\left(y_{p}+x_{f 1} \gamma-y-x_{f 1} \theta\right)^{2}+3 \beta_{1} x_{r 1}\left(y_{p}-x_{r 1} \gamma-y+x_{r 1} \theta\right)^{2} \\ 0 \\ 0 \\ 0 \\ 0 \\ 3 \beta_{1}\left(y_{p}+x_{f 1} \gamma-y-x_{f 1} \theta\right)^{2}+3 \beta_{1}\left(y_{p}-x_{r 1} \gamma-y+x_{r 1} \theta\right)^{2} \\ 3 \beta_{1} x_{f 1}\left(y_{p}+x_{f 1} \gamma-y-x_{f 1} \theta\right)^{2}-3 \beta_{1} x_{r 1}\left(y_{p}-x_{r 1} \gamma-y+x_{r 1} \theta\right)^{2}\end{array}\right]^{T}$

$$
\begin{aligned}
& \frac{\partial g_{8}}{\partial Y}= \\
& {\left[\begin{array}{c}
-3 \beta_{1} x_{f 1}\left(y_{p}+x_{f 1} \gamma-y-x_{f 1} \theta\right)^{2}+3 \beta_{1} x_{r 1}\left(y_{p}-x_{r 1} \gamma-y+x_{r 1} \theta\right)^{2} \\
-3 \beta_{1} x_{f 1}^{2}\left(y_{p}+x_{f 1}-y-x_{f 1} \theta\right)^{2}-3 \beta_{1} x_{r 1}^{2}\left(y_{p}-x_{r 1} \gamma-y+x_{r 1} \theta\right)^{2} \\
0 \\
0 \\
0 \\
0 \\
3 \beta_{1} x_{f 1}\left(y_{p}+x_{f 1} \gamma-y-x_{f 1} \theta\right)^{2}-3 \beta_{1} x_{r 1}\left(y_{p}-x_{r 1} \gamma-y+x_{r 1} \theta\right)^{2} \\
3 \beta_{1} x_{f 1}^{2}\left(y_{p}+x_{f 1} \gamma-y-x_{f 1} \theta\right)^{2}+3 \beta_{1} x_{r 1}^{2}\left(y_{p}-x_{r 1} \gamma-y+x_{r 1} \theta\right)^{2}
\end{array}\right]}
\end{aligned}
$$

The overall linearized equations of motion of the equivalent linear model derived from the nonlinear model, with EOM in Eq. (19), can therefore be expressed as follows:

$$
M \ddot{Y}+C \dot{Y}+\left(K^{*}+K_{e}\right) Y=K^{\prime \prime} X+C^{\prime \prime} \dot{X}
$$

In Eq. (26), $K_{e}=\left[\begin{array}{llllllll}\frac{\partial g_{1}}{\partial Y} & \frac{\partial g_{2}}{\partial Y} & \frac{\partial g_{3}}{\partial Y} & \frac{\partial g_{4}}{\partial Y} & \frac{\partial g_{5}}{\partial Y} & \frac{\partial g_{6}}{\partial Y} & \frac{\partial g_{7}}{\partial Y} & \frac{\partial g_{8}}{\partial Y}\end{array}\right]^{T}$, the elements of this matrix are derived in Eq. (22) to Eq. (25). The rest of the variables in Eq. (26) have already been discussed earlier in this section. The linearized EOM can also be used to compute the ratio between the output and the excitation input for each degree-of-freedom, called as displacement transmissibility. For the linearized model, displacement transmissibility due to external excitation can be computed as:

$$
T=\left[-\omega^{2} M+i \omega C+K^{*}+K_{e}\right]^{-1}[\dot{K}+i \omega \dot{C}] .
$$

In Eq. (27), $T$ is the $8 \times 1$ transmissibility matrix while $K$ and $C$ are $8 \times 1$ matrices resulting from excitation due to the irregularity of the road surface.

For the linear model in Eq. (1), the PSD for each DOF resulting from the excitation input due to road surface irregularity can be computed directly, as shown in Eq. (4). While this cannot be done directly for the nonlinear model, the equivalent linear model in Eq. (26) has been used to iteratively determine the response by using the statistical linearization method. A flowchart of this iterative process is shown in Fig. 4. To start the iterative process, the static load, $P$, is used to compute the initial response, $Y$, shown as $Y_{0}$ in the flowchart in Fig. 4. As can be seen from the flowchart in Fig. 4, the equivalent stiffness matrix, $K_{e}$, is not finalized till the norm of the difference in the covariances of the acceleration spectrum is significantly low. In order to carry out the iterative process, a frequency bandwidth of interest needs to be established. For the purposes of this study, the frequency range of 0 to $40 \mathrm{~Hz}$ has been used. This iterative procedure is expected to yield a unique equivalent linear system. ${ }^{3,20}$ 
Table 1. Linear model—modal analysis results.

\begin{tabular}{||c|c|l||}
\hline $\begin{array}{c}\text { Damped natural } \\
\text { frequency (Hz) }\end{array}$ & Damping ratio & \multicolumn{1}{|c|}{ Mode shape } \\
\hline 1.66 & 0.146 & Sprung mass bounce fully coupled with powertrain bounce and a limited amount of sprung mass pitch \\
3.04 & 0.229 & Sprung mass pitch fully coupled with powertrain pitch \\
12.06 & 0.108 & Rear unsprung mass fore-aft fully coupled with powertrain fore-aft \\
15.45 & 0.259 & Rear unsprung mass hop \\
17.55 & 0.245 & Front unsprung mass hop \\
22.09 & 0.103 & Powertrain pitch coupled with some sprung mass pitch \\
23.69 & 0.106 & Powertrain bounce coupled with some sprung mass bounce \\
603.48 & 0.0005 & Rear unsprung mass fore-aft coupled with a limited amount of powertrain fore-aft \\
\hline
\end{tabular}

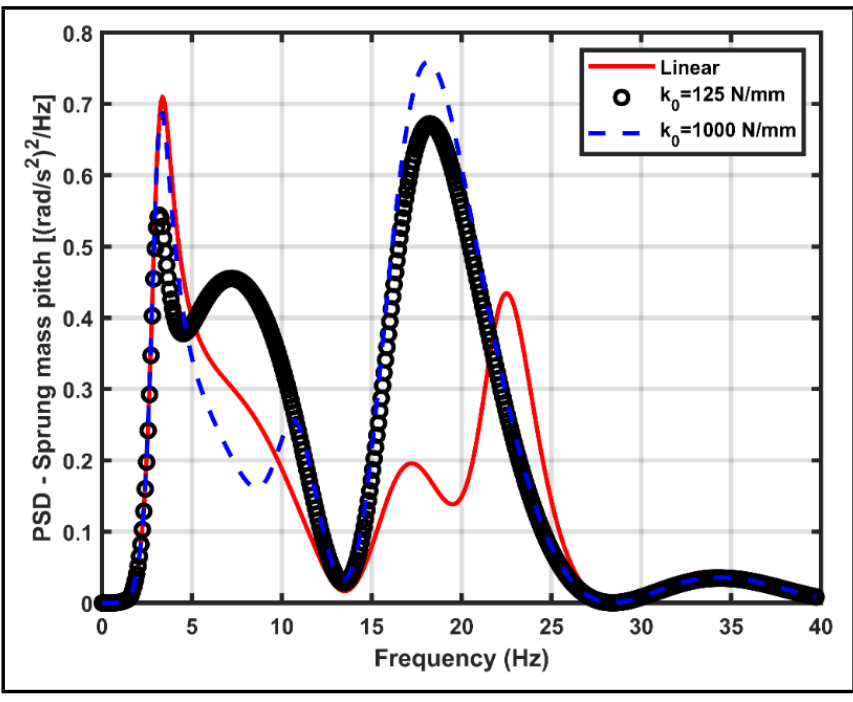

Figure 5. Sprung mass pitch acceleration spectrum at constant speed $(20 \mathrm{~m} / \mathrm{s})$.

The nonlinear model presented in this section has been used for multiple simulations in Section 3. The linear model has been used as a baseline for the results and is used to compare the results of the nonlinear model and to specifically determine the influence of parameters associated with the nonlinear vibration isolation system. All the parameters that have been used for simulation have been identified from relevant literature.

\section{RESULTS}

This section presents the simulation results for the model presented in Section 2. The linear model has been analyzed first to establish a baseline for the nonlinear model. Also, the results of the nonlinear model are analyzed to investigate the influence of different parameters associated with the vibration isolation system. Finally, the results of the model derived from the statistical linearization method are compared with results from numerical integration to evaluate the accuracy of this approach. Most of the motorcycle parameters used for the simulations have been adapted from existing literature while the vibration isolator parameters have been taken from experimental characterization conducted for another study., 8,17 The following values have been used for all the simulations presented in this section: $p=1.4 \mathrm{~m}, b=0.7 \mathrm{~m}, m=200 \mathrm{~kg}$, $I=40 \mathrm{~kg} \mathrm{~m}^{2}, k_{f}=15 \mathrm{kN} / \mathrm{m}, k_{r}=25 \mathrm{kN} / \mathrm{m}, k_{p f}=$ $180 \mathrm{kN} / \mathrm{m}, k_{p r}=180 \mathrm{kN} / \mathrm{m}, m_{f}=15 \mathrm{~kg}, m_{r}=20 \mathrm{~kg}$, $m_{p}=100 \mathrm{~kg}, I_{p}=10 \mathrm{~kg} \mathrm{~m}^{2}, x_{r 1}=320 \mathrm{~mm}, y_{r 1}=55 \mathrm{~mm}$, $x_{f 1}=325 \mathrm{~mm}, y_{f 1}=20 \mathrm{~mm}, x_{s a}=270 \mathrm{~mm}, y_{s a}=30 \mathrm{~mm}$, $k_{1 r x}=350 \mathrm{~N} / \mathrm{mm}, k_{1 f x}=350 \mathrm{~N} / \mathrm{mm}, A=707 \mathrm{~mm}^{2}$, $E=200 \mathrm{GPa}, L=590 \mathrm{~mm}, \phi=5.9^{\circ}$.

The results of the linear model are used as a baseline for the

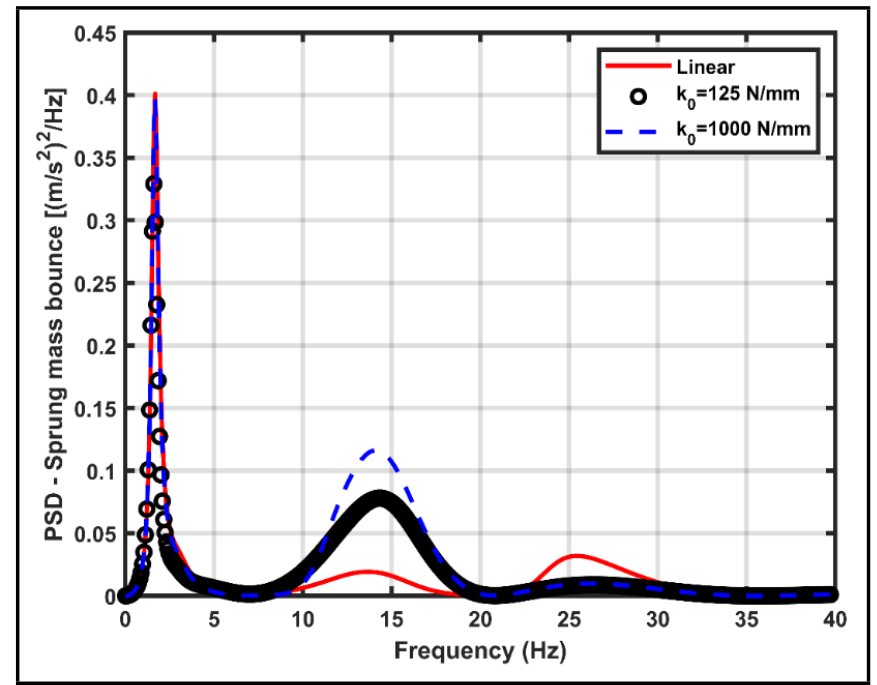

Figure 6. Sprung mass bounce acceleration spectrum at constant speed $(20 \mathrm{~m} / \mathrm{s})$.

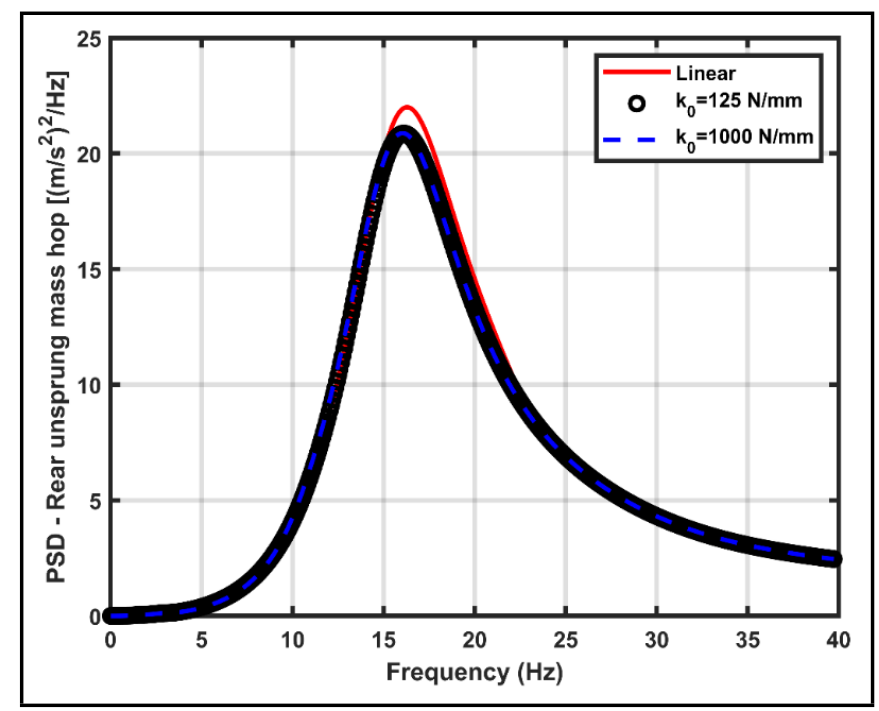

Figure 7. Rear unsprung mass hop acceleration spectrum at constant speed $(20 \mathrm{~m} / \mathrm{s})$.

analysis of the nonlinear model. Table 1 presents a summary of the results from the modal analysis of the linear model. As can be seen from these results in Table 1, the two lowest modes exhibit coupling between sprung mass bounce and sprung mass pitch and coupling of these two modes with the motion of the powertrain.

It can also be seen from the results in Table 1 that the fore-aft mode of the powertrain is coupled to the rear unsprung mass due to the swing arm connection. Furthermore, the rear and front unsprung masses exhibit uncoupled hop modes with a relatively higher damping ratio. Finally, the highest mode is 


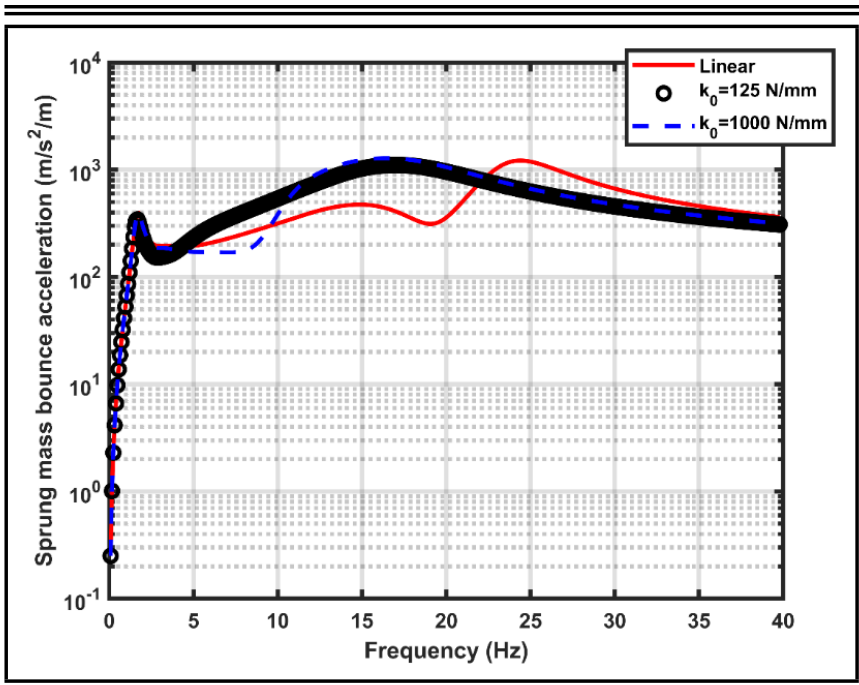

Figure 8. Acceleration response-sprung mass bounce.

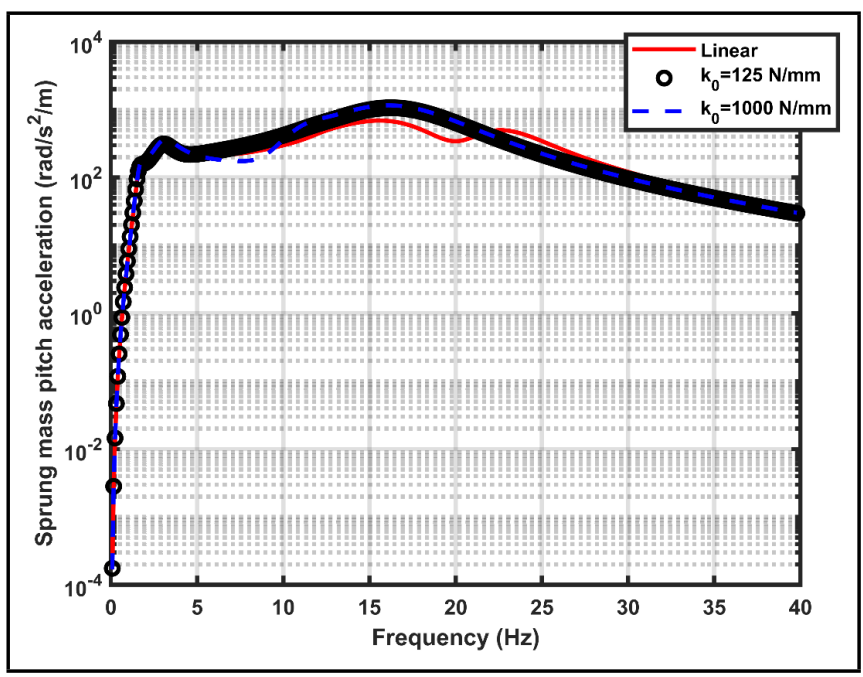

Figure 9. Acceleration response—-sprung mass pitch.

primarily due to the highly stiff swing arm connecting the rear unsprung mass to the powertrain, this mode is well outside the frequency range of interest for the purposes of this study and will not be used for any analysis. The results of the linear model are comparable to other similar models in the literature, specifically for the modes related to the sprung mass and the unsprung masses. ${ }^{17}$

While the use of the nonlinear vibration isolation system is not seen to influence the acceleration spectrum of the unsprung masses significantly, it can be clearly seen to influence the acceleration spectrum of the sprung mass, as seen from the results in Fig. 5 and Fig. 6. It can be seen that the peak of the bounce acceleration spectrum as well as the pitch acceleration spectrum of the sprung mass reduces in the low frequency range while there is a corresponding increase in the peak and bandwidth of the sprung mass spectrum at higher frequencies (ranging from 10 to $20 \mathrm{~Hz}$ ). This increase can be mitigated somewhat by lowering the vertical stiffness of the nonlinear isolators $\left(k_{0}\right)$ to maximize the benefits of the HSLDS design of a vibration isolator. Although the pitch acceleration spectrum of the sprung mass exhibits a reasonable reduction in the lower frequency range, the peak in the higher frequency range (10 to $20 \mathrm{~Hz}$ ) does not reduce substantially with decreasing values of

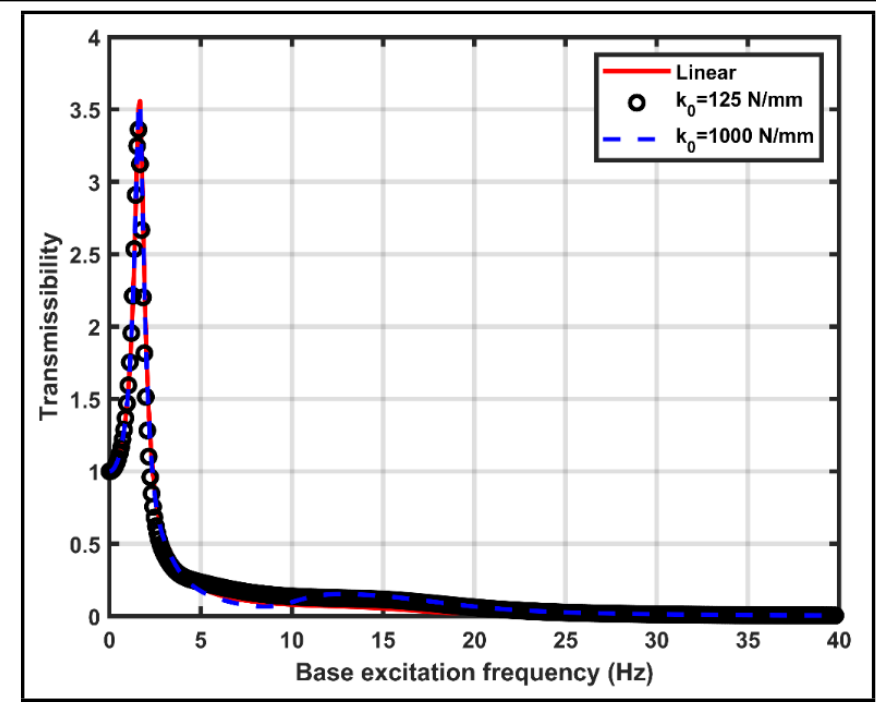

Figure 10. Displacement transmissibility—sprung mass bounce.

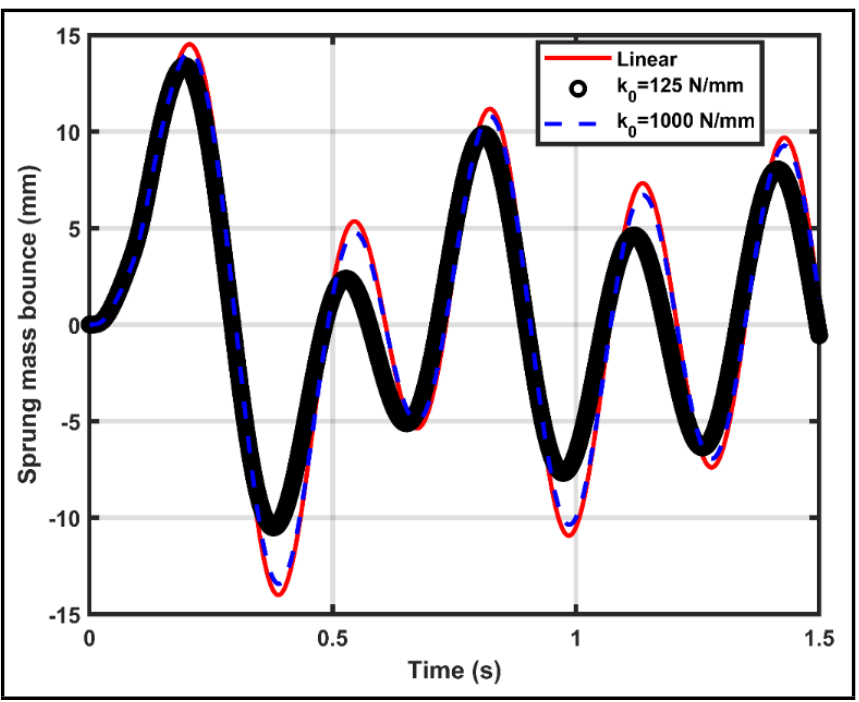

Figure 11. Time domain response—-sprung mass bounce.

$k_{0}$, as clearly seen in Fig. 6 and to some extent in Fig. 5. This could be attributed to the powertrain modes in this frequency range that are directly coupled to the sprung mass. It may be noted that the reduction of the sprung mass spectrum is not achieved at the expense of the rear unsprung mass hop, as seen from the results in Fig. 7. This implies that the HSLDS design could be used to mitigate the trade-off between ride comfort and handling. It is observed that the spectrum of the rear unsprung mass hop acceleration shows very little sensitivity to the vertical stiffness of the nonlinear vibration isolators, and is not significantly different from the system with the linear vibration isolation system. This can be primarily attributed to the independent hop modes of the two unsprung masses. Furthermore, this result indicates that the nonlinear vibration isolators can be designed to marginally improve ride comfort in the lower frequency range without any adverse impact on handling.

Acceleration response is widely used in vibration analysis for the evaluation of ride comfort. For the planar motorcycle model, observations from the acceleration response are somewhat similar to the results from power spectral density. It can be seen from Fig. 8 and Fig. 9 that acceleration of the sprung 


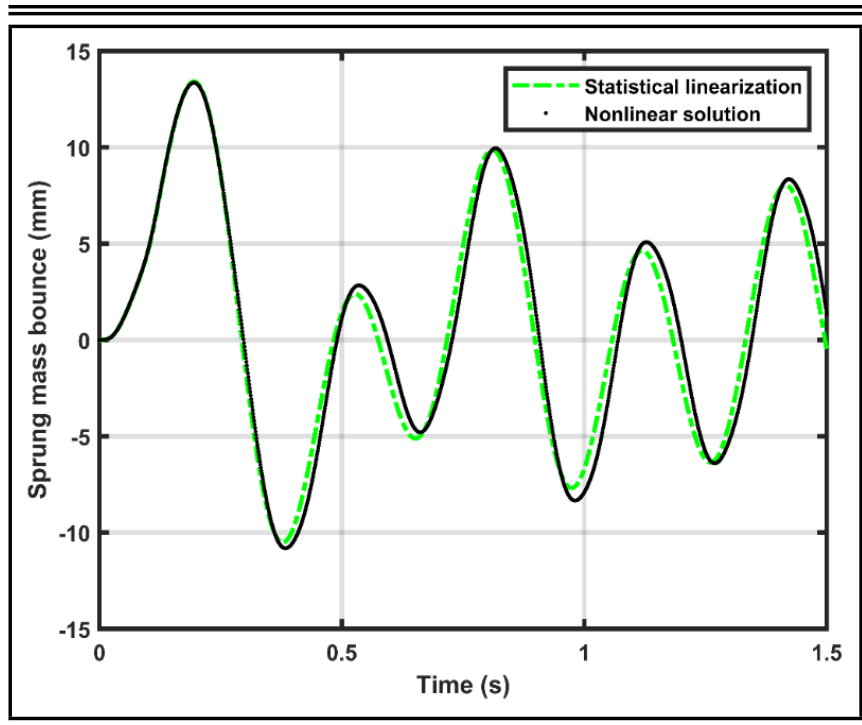

Figure 12. Time domain response-comparison—-sprung mass bounce.

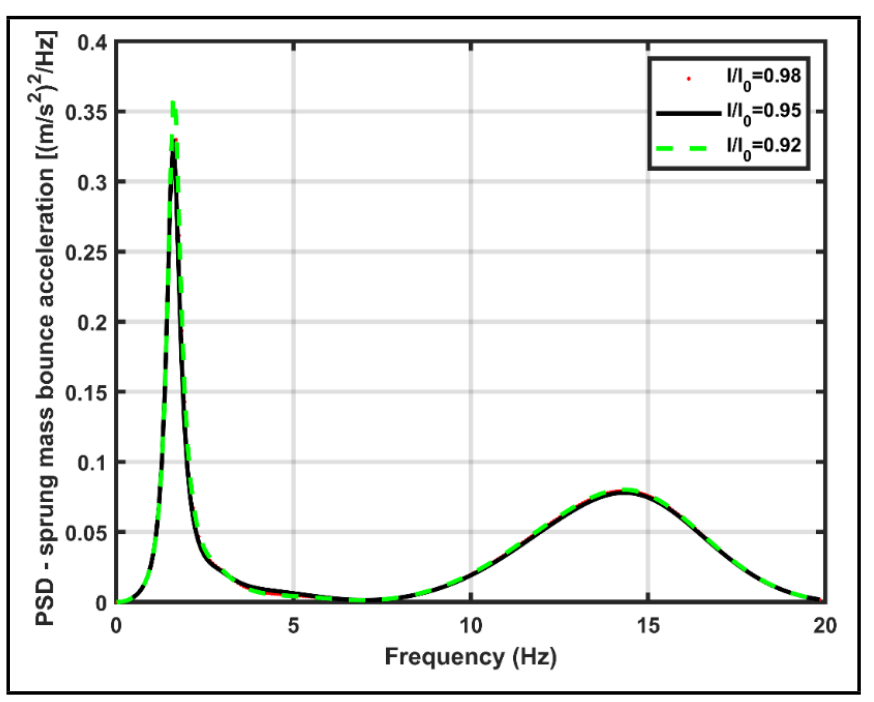

Figure 13. Sprung mass bounce acceleration spectrum at constant speed $(20 \mathrm{~m} / \mathrm{s})$ - influence of pre-compression.

mass is either lower or identical to the system with linear vibration isolators except for the frequency range of 10 to $20 \mathrm{~Hz}$. Furthermore, displacement transmissibility results in Fig. 10 show that the peak transmissibility of the sprung mass bounce reduces marginally with the use of the nonlinear vibration isolation system. It is observed that the acceleration response as well as displacement transmissibility of the unsprung masses is not affected much by using the nonlinear vibration isolation system. This implies that the use of the nonlinear vibration isolation system does not seem to have an adverse impact on handling. The transmissibility results can be further substantiated from the response of the sprung mass in the time domain while the motorcycle is traveling in a straight line at a constant speed of $20 \mathrm{~m} / \mathrm{s}$ (72 kph) over a bumpy road that has a sinusoidal profile with an amplitude of $20 \mathrm{~mm}$ and a wavelength of $6 \mathrm{~m}$. Results for the time history of sprung mass bounce can be seen in Fig. 11, and it is seen that the amplitude reduces slightly with the use of the nonlinear vibration isolation system while remaining sensitive to the design parameters.

In order to evaluate the accuracy of the response resulting from the statistical linearization method, a comparison of the

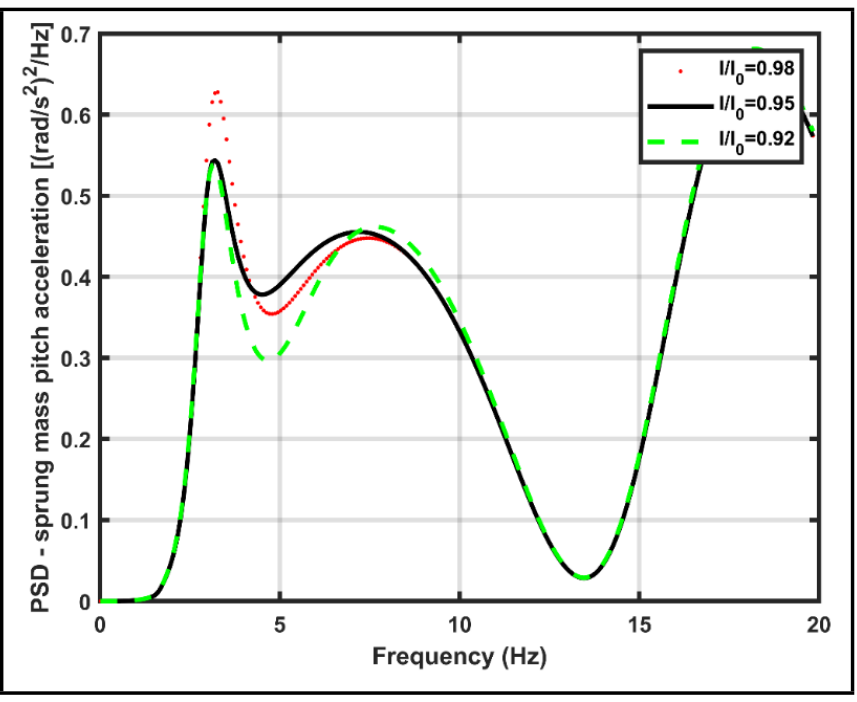

Figure 14. Sprung mass pitch acceleration spectrum at constant speed $(20 \mathrm{~m} / \mathrm{s})$ - influence of pre-compression.

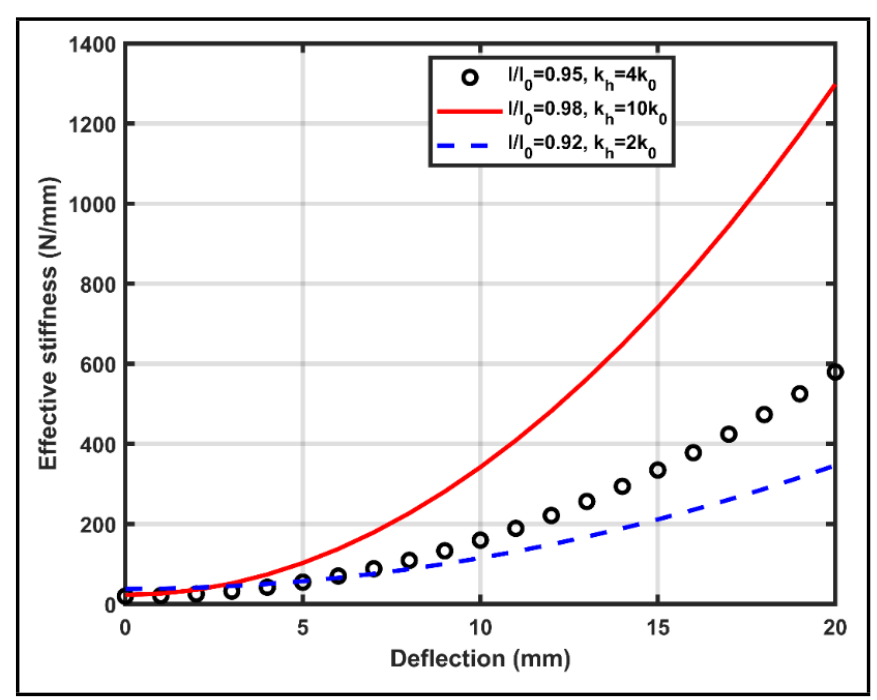

Figure 15. Nonlinear stiffness characteristics-influence of pre-compression.

results for the sprung mass bounce is shown in Fig. 12. It can be seen that the time domain response from the statistical linearization method is within $1 \%$ of the results from numerical integration of the nonlinear model. The comparison of the time history of other degrees-of-freedom exhibits similar results and is not being shown here. A single step solution with an explicit Runge-Kutta formulation has been used for numerical integration. ${ }^{21}$ It can, therefore, be concluded that statistical linearization can be reliably used for analyzing the nonlinear model presented in this study. This method is robust and numerically efficient, as indicated in the reference literature. ${ }^{20}$ Additional nonlinearities associated with the system could also be investigated by using the statistical linearization method.

In order to demonstrate the full range of results from the use of the nonlinear design of the vibration isolation system with HSLDS characteristics, a few more simulations have been performed to specifically investigate parameter sensitivity under multiple operating conditions. Figure 13 and Fig. 14 show the influence of varying levels of pre-compression of the nonlinear vibration isolators on the acceleration spectrum of the sprung mass. While a change in the pre-compression of the nonlinear vibration isolators is seen to only slightly influence 


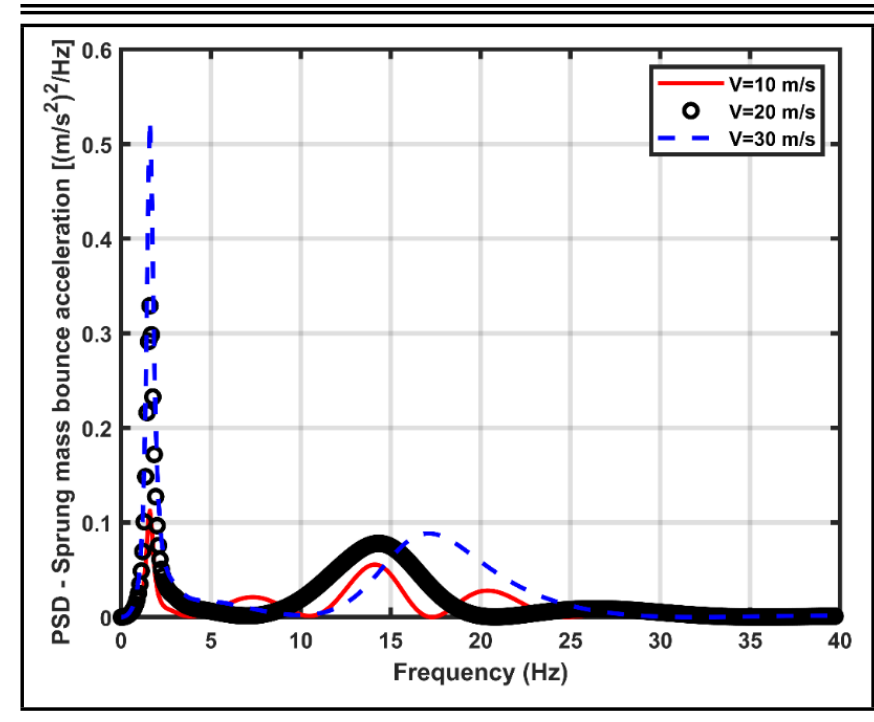

Figure 16. Sprung mass bounce acceleration spectrum-influence of speed.

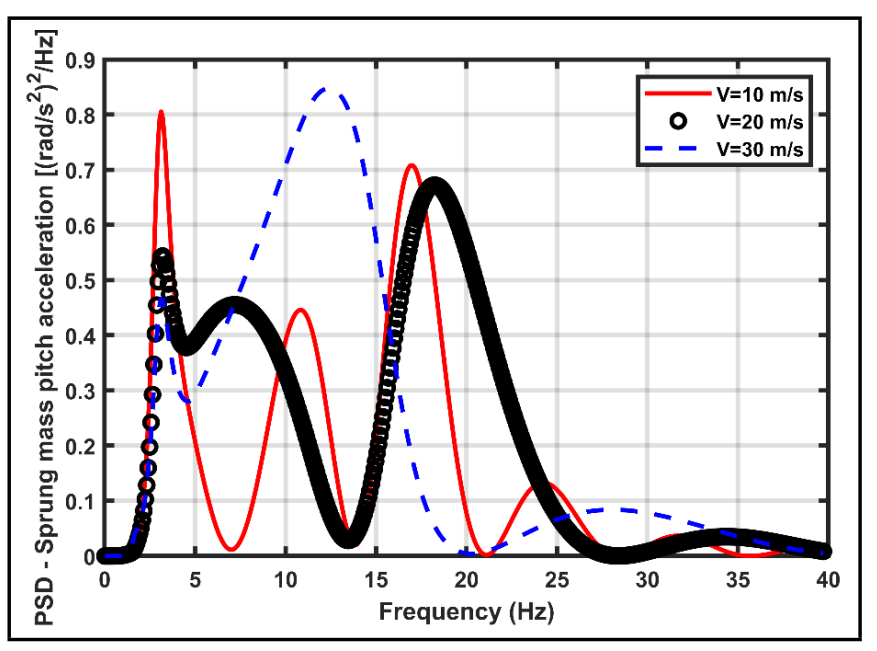

Figure 17. Sprung mass pitch acceleration spectrum—influence of speed.

the peak of the bounce acceleration spectrum of the sprung masses, it is seen to influence the peak as well as the bandwidth of the pitch acceleration spectrum. This could be attributed to the relationship between pre-compression and effective stiffness of the vibration isolation system. These results indicate that pre-compression of the HSLDS design can be used to fine tune ride comfort. It should be noted that a change in precompression does not influence the acceleration spectrum of unsprung masses, therefore this parameter is not expected to have an influence on handling. The direct influence of precompression on the effective stiffness of the nonlinear vibration isolators can be seen from Fig. 15. It may be noted that the choice of pre-compression is directly related to the out-ofplane stiffness due to the stability criterion, therefore exercising an influence on the spatial dynamics of the motorcycle. ${ }^{17}$

Spectral analysis and time domain analysis is performed at three different speeds to observe any trends as the speed of the motorcycle increases from $10 \mathrm{~m} / \mathrm{s}(36 \mathrm{kph})$ to $20 \mathrm{~m} / \mathrm{s}$ (72 kph), and eventually to $30 \mathrm{~m} / \mathrm{s}(108 \mathrm{kph})$. It may be noted that the spectral analysis is carried out while the bike is traveling over an asphalted road that is considered as a good road surface as per the classification of the International Organization for Standardization. ${ }^{19}$ Three different speeds have been chosen to encompass a wide range of speeds that the bike may be trav-

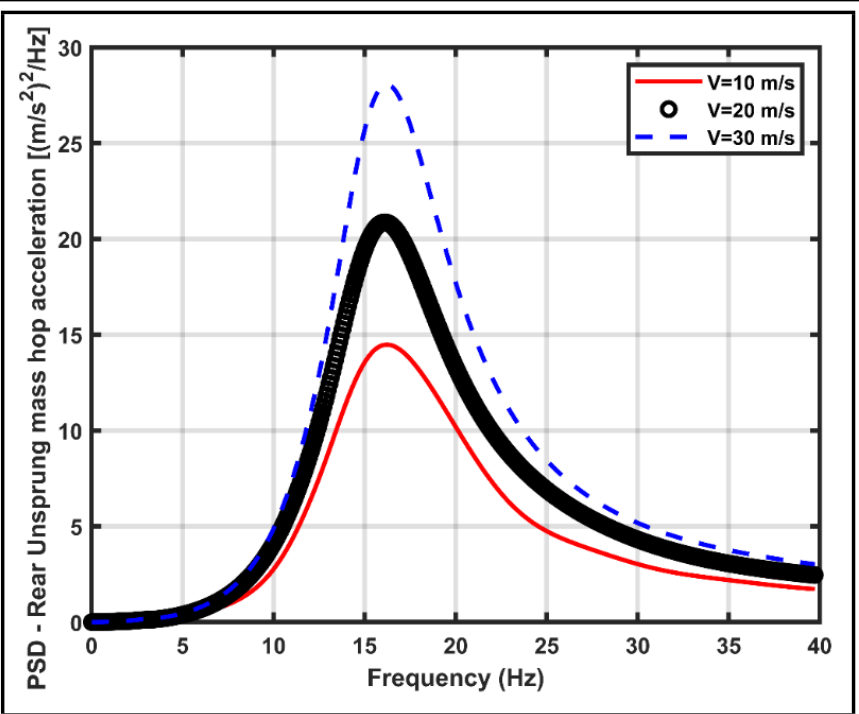

Figure 18. Rear unsprung mass hop acceleration spectrum-influence of speed.

eling. It can be seen from Fig. 16 and Fig. 17 that although the spectrum for bounce acceleration exhibits a significant increase in the peak with an increasing speed, the results for the pitch acceleration spectrum are complex and do not show a direct trend with increasing speed. This could be due to the significant coupling between the bounce and pitch modes of the sprung mass. On the other hand, the spectrum of the unsprung masses exhibits a direct increase in the peak as well as bandwidth with an increasing speed, an example of such a result can be seen from the spectrum of the rear unsprung hop acceleration in Fig. 18. It can be observed from Fig. 18 that the frequency corresponding to the peak spectrum does not vary much as the speed increases from $10 \mathrm{~m} / \mathrm{s}$ to $30 \mathrm{~m} / \mathrm{s}$, this is primarily because the excitation frequencies in this speed range are much lower than the natural modes of the unsprung mass.

Results from the time domain analysis can be seen in Fig. 19 and Fig. 20. The time domain analysis is performed when the motorcycle is traveling in a straight line with a constant speed over a sinusoidal bump course with an amplitude of $20 \mathrm{~mm}$ and a wavelength of $6 \mathrm{~m}$. This corresponds to an excitation frequency of $1.67 \mathrm{~Hz}, 3.33 \mathrm{~Hz}$, and $5 \mathrm{~Hz}$ for a speed of $10 \mathrm{~m} / \mathrm{s}, 20 \mathrm{~m} / \mathrm{s}$, and $30 \mathrm{~m} / \mathrm{s}$ respectively. Wheelbase filtering has been used such that the displacement input at the rear tire is $Y_{r}=y \sin \left(2 \pi \frac{V}{L} t-2 \pi \frac{p}{L}\right)$ while the displacement input at the front tire is $Y_{f}=y \sin \left(2 \pi \frac{V}{L} t\right)$, where $V$ is the speed of the bike, $p$ is the wheelbase, $L$ is the wavelength of the bump course, $y$ is the amplitude of the bump course, and $t$ is time. The results for sprung mass bounce from Fig. 19 indicate that a reduction in speed results in an increase in the amplitude of the output while an increase in speed results in an increase in the frequency of the response. This can be directly attributed to the natural mode of the sprung mass that is being primarily excited at a speed of $10 \mathrm{~m} / \mathrm{s}$, and the excitation frequency shifting away from the bounce mode with an increase in speed. The sprung mass pitch response in Fig. 20 does not show a direct trend with increasing speed like the sprung mass bounce, this could be due to the natural mode corresponding to the sprung mass pitch being specifically excited at $20 \mathrm{~m} / \mathrm{s}$ as well as the coupling between the pitch and bounce motion of the sprung mass. 


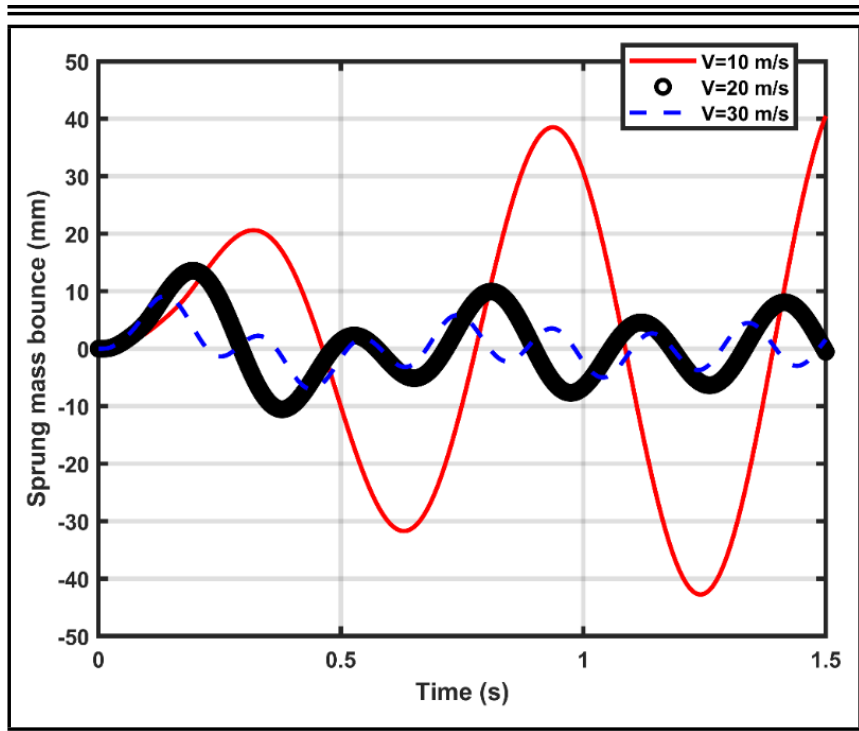

Figure 19. Time domain response — sprung mass bounce over bump courseinfluence of speed.

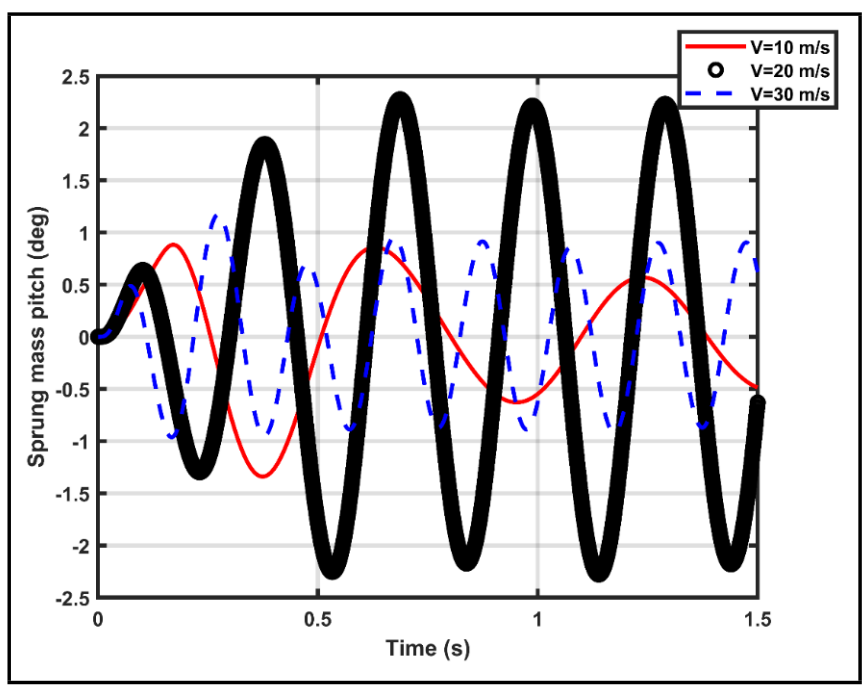

Figure 20. Time domain response—-sprung mass pitch over bump courseinfluence of speed.

Finally, the influence of the wavelength of the sinusoidal bump course can be seen in Fig. 21 and Fig. 22 while the motorcycle is traveling in a straight line at a constant speed of $20 \mathrm{~m} / \mathrm{s}$ over a sinusoidal bump course with an amplitude of $20 \mathrm{~mm}$. As the wavelength $(\lambda)$ of the bump course changes from $2 \mathrm{~m}$ to $3 \mathrm{~m}$ to $5 \mathrm{~m}$, the excitation frequency reduces from $10 \mathrm{~Hz}$ to $6.67 \mathrm{~Hz}$ to $4 \mathrm{~Hz}$ respectively. It can be observed that the sprung mass bounce amplitude is significantly higher at $\lambda=5 \mathrm{~m}$ since the natural mode corresponding to this DOF gets predominantly excited at this wavelength, as seen from the results in Fig. 21. This can be explained from the list of natural modes of the linear model in Table 1, the lowest natural mode pertains to the sprung mass and is close to the excitation frequency corresponding to the wavelength of $\lambda=5 \mathrm{~m}$. Similarly, it can be seen from the sprung mass pitch in Fig. 22 that the amplitude is reasonably higher for $\lambda=5 \mathrm{~m}$ due to the sprung mass pitch mode and the coupling between the sprung mass bounce and pitch. Such an analysis can be specifically beneficial for evaluating parameter sensitivity of the vibration isolation system before starting detailed design.

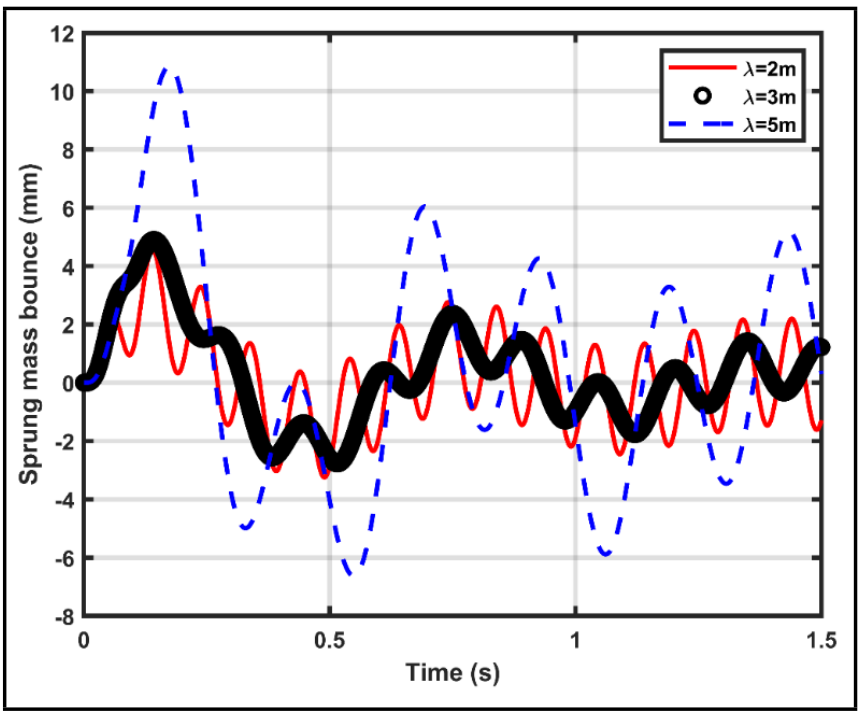

Figure 21. Time domain respons—sprung mass bounce-influence of bump course wavelength.

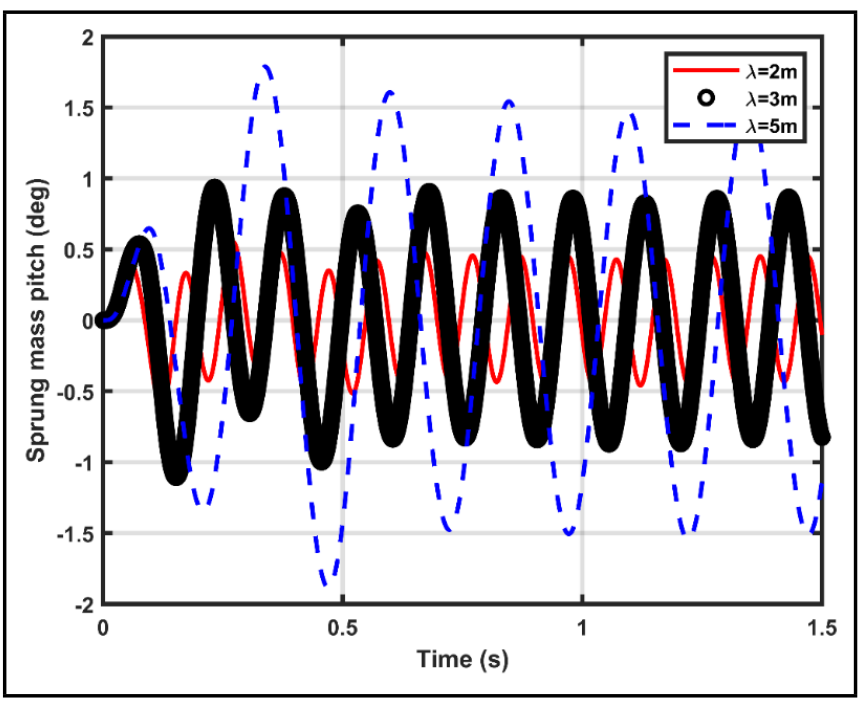

Figure 22. Time domain response—sprung mass pitch—influence of bump course wavelength.

\section{CONCLUSIONS}

A comprehensive model has been presented in this paper that can be used to analyze the planar dynamics of a motorcycle with a specific focus on evaluating the influence of nonlinearities of the vibration isolation system. Although this paper discusses one specific form of nonlinearity of the vibration isolation system, the general approach used in this study could be applicable to the investigation of other nonlinearities associated with the planar dynamics involving the front and rear suspension systems.

Results indicate that ride comfort can be improved in the low frequency range by using the nonlinear vibration isolation system without any adverse impact on handling. This is particularly observed in the relatively lower peaks of the acceleration spectrum of the sprung mass in the frequency range of 1 to $6 \mathrm{~Hz}$. Since this frequency range is considered to be significant for ride comfort of the upper body of the rider, the nonlinear vibration isolation system is expected to be particularly beneficial if the associated parameters are specifically tuned for ride comfort. Furthermore, the nonlinear vibration isolation 
system allows design flexibility to vary the pre-compression as well as out-of-plane stiffness to meet the needs of spatial dynamics of the motorcycle. The one disadvantage of the nonlinear vibration isolation system observed from the results of this study is the relative increase in the acceleration spectrum of the sprung mass and its bandwidth in the frequency range of 10 to $20 \mathrm{~Hz}$. This frequency range is considered to be important for ride comfort of the rider's arms and hands, and not the entire upper body. ${ }^{22}$ This disadvantage could be mitigated to some extent by using some form of local vibration isolation at the handlebars. ${ }^{23}$

The main benefit of the model and analysis presented in this paper is that ride comfort and handling can be analyzed to evaluate the influence of the coupled dynamics of the rear suspension system and the vibration isolation system in the presence of nonlinearities. This could allow an analyst to evaluate specific nonlinear attributes of the suspension system as well as the vibration isolator design before starting detailed design of individual components. Future work will aim to investigate the influence of stiffness and damping nonlinearities in alternate rear suspension designs on the planar dynamics of a motorcycle. An experimental evaluation of the findings of this study will also be pursued in the future.

\section{REFERENCES}

1 Mechanical Simulation Corp, BikeSim, Ann Arbor, MI, USA, (2017).

2 Dynamotion Srl, FastBike, Padova, Italy, (2017).

3 Ricardo, L. A. R. Nonlinear Stochastic Analysis of Motorcycle Dynamics, PhD Thesis, Houston, TX, USA, Rice University, (2013).

4 Ibrahim, R. A. Recent Advances in Nonlinear Passive Vibration Isolators, Journal of Sound and Vibration, 314, 371-452, (2008). https://dx.doi.org/10.1016/j.jsv.2008.01.014

${ }^{5}$ Lu, Z., Brennan, M. J., Yang, T., Li, X., and Liu, Z. An investigation of a two-stage nonlinear vibration isolation system, Journal of Sound and Vibration, 332, 1456-1464, (2013). https://dx.doi.org/10.1016/j.jsv.2012.11.019

6 Shaw, A. D., Neild, S. A., and Wagg, D. J. Dynamic analysis of high static low dynamic stiffness vibration isolation mounts, Journal of Sound and Vibration, 332, 1437-1455, (2013). https://dx.doi.org/10.1016/j.jsv.2012.10.036

7 Lu, Z., Gu, D., Ding, H., Lacarbonara, W., and Chen, L. Nonlinear vibration isolation via a circular ring, Mechanical Systems and Signal Processing, 136, 106490, (2020). https://dx.doi.org/10.1016/j.ymssp.2019.106490

8 Kaul, S. Nonlinear design of a passive vibration isolator: influence of multi-axial stiffness, Proceedings of the ASME 2019 International Mechanical Engineering Congress and Exposition, IMECE 2019-10021, Salt Lake City, UT, USA, (2019). https://dx.doi.org/10.1115/IMECE2019-10021

9 Peng, Z. K., Meng, G., Lang, Z. Q., Zhang, W. M., and Chu, F. L. Study of the effects of cubic nonlinear damping on vibration isolations using Harmonic Balance Method, International Journal of Non-Linear Mechanics, 47, 1073-1080, (2012) https://dx.doi.org/10.1016/j.ijnonlinmec.2011.09.013

10 Richards, C. M. and Singh, R. Characterization of rubber isolator nonlinearities in the context of singleand multi-degree-of-freedom experimental systems, Journal of Sound and Vibration, 247, 807-834, (2001). https://dx.doi.org/10.1006/jsvi.2001.3759

11 Tang, B. and Brennan, M. J. A comparison of two nonlinear damping mechanisms in a vibration isolator, Journal of Sound and Vibration, 332, 510-520, (2013). https://dx.doi.org/10.1016/j.jsv.2012.09.010

$12 \mathrm{Xu}, \mathrm{Z}$. D., Liao, Y. X., Ge, T., and Xu, C. Experimental and theoretical study of viscoelastic dampers with different matrix rubbers, Journal of Engineering Mechanics, 142, 04016051-1-12, (2016). https://dx.doi.org/10.1061/(ASCE)EM.19437889.0001101

13 Alonso, A., Gil-Negrete, N., Nieto, J., and Gimenez, J. G. Development of a rubber component model suitable for being implemented in railway dynamic simulation programs, Journal of Sound and Vibration, 332, 3032-3048, (2013). https://dx.doi.org/10.1016/j.jsv.2013.01.002

14 Kaul, S. Dynamic modeling and analysis of mechanical snubbing, Journal of Vibration and Acoustics, 134, 0210201-8, (2012). https://dx.doi.org/10.1115/1.4005012

15 Kaul, S. Influence of a vibration isolation system on planar dynamics of a motorcycle, International Journal of Acoustics and Vibration, 25, 96-103, (2020). https://dx.doi.org/10.20855/ijav.2020.25.11603

16 Carrella, A. Passive Vibration Isolators with High-StaticLow-Dynamic-Stiffness, VDM Verlag, Germany, (2010).

17 Cossalter, V. Motorcycle Dynamics, Second Edition, Lulu, Morrisville, NC, USA, (2006).

18 Cocco, G. Motorcycle Design and Technology, Giorgio Nada, Vimodrone, Italy, (2001).

19 Sun, L. On human perception and evaluation to road surfaces, Journal of Sound and Vibration, 247, 547-560, (2001). https://dx.doi.org/10.1006/jsvi.2001.3642

20 Roberts, J. B. and Spanos, P. D. Random Vibration and Statistical Linearization, Dover Publications, Mineola, NY, USA, (2003).

21 Shampine, L. F. and Reichelt, M. W. The MATLAB ODE Suite, SIAM Journal on Scientific Computing, 18, 1-22, (1997). https://dx.doi.org/10.1137/S1064827594276424

22 Cossalter, V., Doria, A., Garbin, S., and Lot, R. Frequencydomain method for evaluating the ride comfort of a motorcycle, Vehicle System Dynamics, 44, 339-355, (2006). https://dx.doi.org/10.1080/00423110500420712

23 Agostoni, S., Cheli, F., Leo, E., and Pezzola, M. An innovative multi dof TMD system for motorcycle handlebars designed to reduce structural vibrations and human exposure, Mechanical Systems and Signal Processing, 31, 298-315, (2012). https://dx.doi.org/10.1016/j.ymssp.2011.11.018 